\title{
A Review of Microfiber and Nanofiber Based Optical Sensors
}

\author{
George Y. Chen, Ming Ding, Trevor. P. Newson and Gilberto Brambilla*
}

Optoelectronics Research Centre, University of Southampton, Southampton, SO17 1BJ, U.K

\begin{abstract}
Rapid advances in optical microfiber and nanofibers (MNF) based sensors have been driven by powerful industries such as automotive, biomedical and defense, with the increasing demand for highly-sensitive, selective, nonintrusive, fast-response, compact and robust sensors that can perform in-situ measurements at remote and harsh environments. A diverse range of MNF based sensors have been developed for measuring refractive index, bio-chemical, temperature, current, displacement, bend, surface, acceleration, force, rotation, acoustic, electric field and magnetic field. Given the growing interest for this exciting area of scientific research, new designs are emerging continuously at a fast pace. This paper attempts to provide a comprehensive review of all MNF based sensors reported to-date. Sensors are divided according to their morphology and measurand.
\end{abstract}

Keywords: Microfiber, microwire, nanofiber, nanowire, taper, optical, sensor, sensing, review.

\section{INTRODUCTION}

Optical microfibers and nanofibers (MNF), also known as optical/photonic microwires and nanowires, are optical fiber tapers with a uniform waist region size comparable to the wavelength. MNFs are usually manufactured by heating and stretching [1] regular-sized optical fibers. The result is a biconical taper that provides a smooth lossless connection to other fiberized components. By controlling the pulling rate during the fabrication process, the taper profile can be finetuned to suit the application [2,3]. Optical materials other than silica have been used to manufacture MNFs, including phosphate [4], tellurite [4], lead silicate [5], bismuthate [5] and chalcogenide glasses [6] and a variety of polymers [711]. The remarkable optical and mechanical properties exhibited by MNFs are constantly exploited for optical sensing and include large evanescent fields, strong optical confinement, flexibility, configurability and robustness. Such desirable characteristics have gathered much attention in recent years and it is what makes MNFs an excellent platform for optical sensors. In the next section, the unique waveguiding properties of MNFs will be discussed, followed by an explanation of the general parameters of a sensor; and lastly, an overview of both non-resonator-type and resonator-type MNF based sensors.

\section{OPTICAL WAVEGUIDING PROPERTIES OF MNFS}

Light launched into a MNF will propagate as core modes which are defined to be the state when their effective index lies between the cladding and core indices. The effective index decreases monotonically along the down-taper transition, and the mode becomes guided by the cladding/air

*Address correspondence to this author at the Optoelectronics Research Centre, University of Southampton, Southampton, SO17 1BJ, U.K; Tel: +44 238059 3588; Fax: +44 238059 3149;

E-mail: gb2@orc.soton.ac.uk interface. To analyze the mode propagation in the MNF, the exact solution of the Maxwell equations needs to be found [12] since the weakly guiding approximation is not valid as a result of the large index difference between the cladding and air:

$$
\begin{aligned}
& {\left[\frac{J_{v}^{\prime}(U)}{U J_{v}(U)}+\frac{K_{v}^{\prime}(U)}{W K_{v}(U)}\right]\left[\frac{J_{v}^{\prime}(U)}{U J_{v}(U)}+\left(\frac{n_{\text {surr }}}{n_{M N F}}\right)^{2} \frac{K_{v}^{\prime}(U)}{W K_{v}(U)}\right]=} \\
& v^{2}\left(\frac{1}{U^{2}}+\frac{1}{W^{2}}\right)\left(\frac{1}{U^{2}}+\left(\frac{n_{\text {surr }}}{n_{M N F}}\right)^{2} \frac{1}{W^{2}}\right)
\end{aligned}
$$

where $\quad U=r \sqrt{k_{0}{ }^{2} n_{M N F}{ }^{2}-\beta^{2}}, \quad W=r \sqrt{\beta^{2}-k_{0}{ }^{2} n_{\text {surr }}{ }^{2}}$, $J_{v}$ is the $v^{\text {th }}$ order Bessel function of the first kind, $K_{v}$ is the $v^{\text {th }}$ order modified Bessel function of the second kind, $n_{M N F}$ and $n_{\text {surr }}$ are the refractive indices of the MNF ( 1.45) and the surrounding medium. $\beta$ is the effective propagation constant of the optical mode. $r$ is used to denote the core radius and correspond to the center from the core-cladding interface in solid fibers and to the cladding-air interface in MNFs. The propagation constant of hybrid modes can be calculated by solving Eq. 1 .

The V number can be expressed as:

$V=\sqrt{U^{2}+W^{2}}=\frac{2 \pi}{\lambda} \cdot r \cdot \mathrm{NA}$

As it is in optical fibers, MNFs experience single-mode operation when $\mathrm{V}<2.405$ [13].

\subsection{Optical Confinement}

MNFs can confine light to the diffraction limit for lengths that are only limited by loss and are usually several orders of magnitude longer than the typical Rayleigh length. 


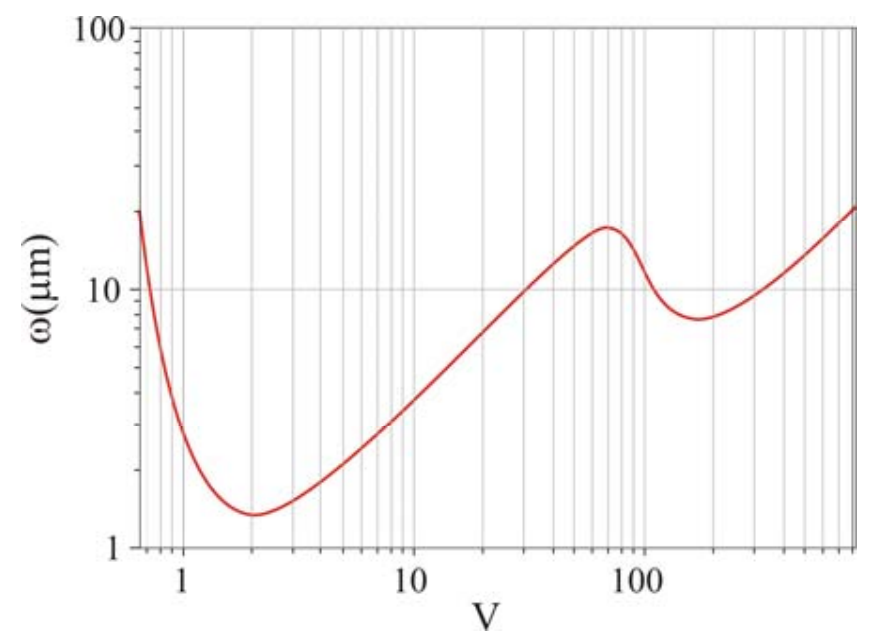

Fig. (1). Dependence of the spot size on the V number of a MNF. The two troughs represent the points of maximum confinement in the cladding/core.

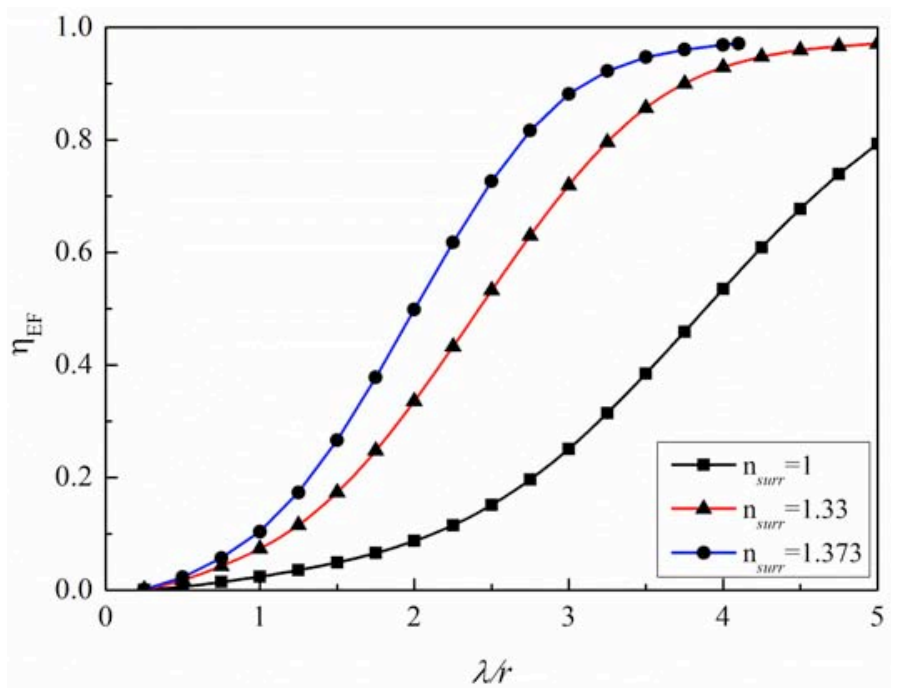

Fig. (2). Dependence of the power fraction propagating in the evanescent field $\eta_{E F}$ on the normalised wavelength $\lambda / r$ in different refractive index surroundings.

As shown in Fig. (1), when the optical fiber diameter decreases, the $\mathrm{V}$ number and spot size $\omega$ initially decreases until the point of maximum confinement within the core. After that, the mode is no longer guided in the core and $\omega$ suddenly increases to a maximum associated with cladding guiding. For even smaller diameters, $\omega$ decreases with decreasing $\mathrm{V}$ until it reaches a minimum around $\mathrm{V} \sim 2$, before increasing again. The region below $\mathrm{V}<2$ is typical of MNFs: the mode is only weakly guided by the waveguide, so $\omega$ can be orders of magnitude larger than the physical diameter of the MNF and thus a larger fraction of the power resides in the evanescent field. For $\mathrm{V}<0.6$, the beam spot size can continue to expand until it becomes orders of magnitude larger than $r$.

\subsection{Evanescent Field}

When $\mathrm{V} \ll<1$, a large portion of the power propagates as the evanescent field outside the MNF. The extension of the evanescent field and the fraction of power $\eta_{E F}$ propagating in it depend on the ratio $\lambda / r$ and can be obtained from the com- ponent of the pointing vector along the direction of the beam propagation $S_{z}$ [13].

$\eta_{E F}=\frac{\int_{\text {out }} S_{z} d A}{\int_{\text {in }} S_{z} d A+\int_{\text {out }} S_{z} d A}=\frac{\int_{r}^{\infty} S_{z} d A}{\int_{o}^{r} S_{z} d A+\int_{r}^{\infty} S_{z} d A}$

where $\int_{\text {in }} S_{z} d A$ and $\int_{\text {out }} S_{z} d A$ are the integrals of $S_{z}$ over the MNF cross section and outside the MNF respectively. Fig. (2) shows the dependence of $\eta_{E F}$ on the normalized wavelength $\lambda / r$ for silica MNFs. When the surrounding medium is air, $\eta_{E F}$ reaches 0.5 at $\lambda / r \sim 4$, meaning that half of the power is propagating outside the MNF when the MNF radius is a quarter of the wavelength of light propagating in it. $\eta_{E F}$ increases for increasing surrounding refractive indices when the normalized wavelength $\lambda / r$ is kept constant. For example, for a surrounding refractive index of $n_{\text {surr }}=1.373$, more than $90 \%$ power lies in the evanescent field when $\lambda / r \sim 4$. Therefore, to enhance the evanescent field, polymers or Teflon can be used to embed the MNF. To improve the $\eta_{E F}$ even further, 
(a)

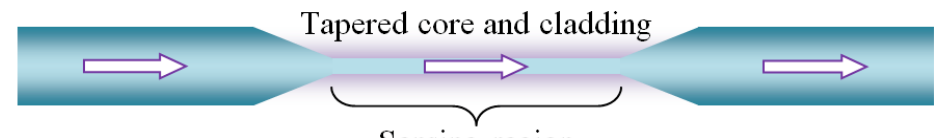

Sensing region

Evanescently coupled MNF

(b)

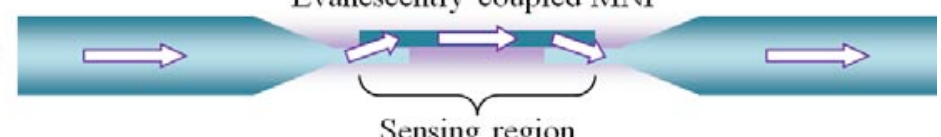

Sensing region

(c)

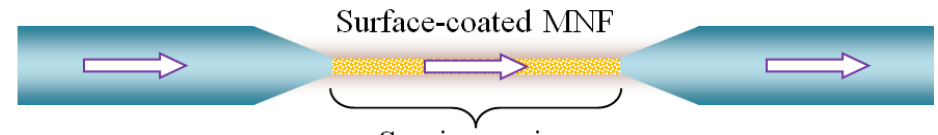

Sensing region

(d)

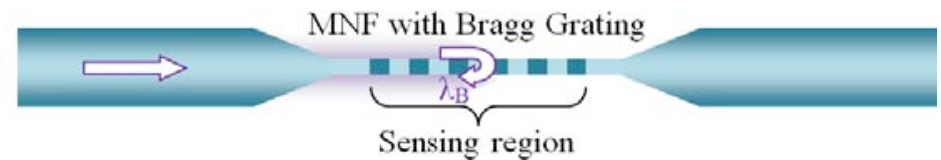

MNF based MZ-interferometer

(e)

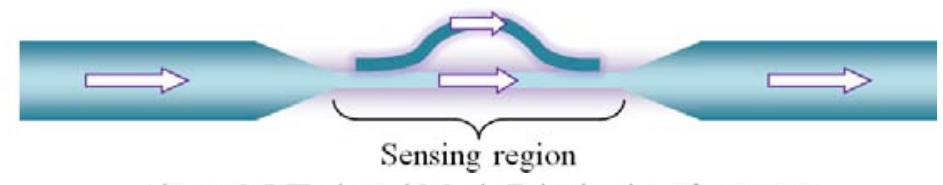

Abrupt-MNFs based Mach-Zehnder interferometer

(f)

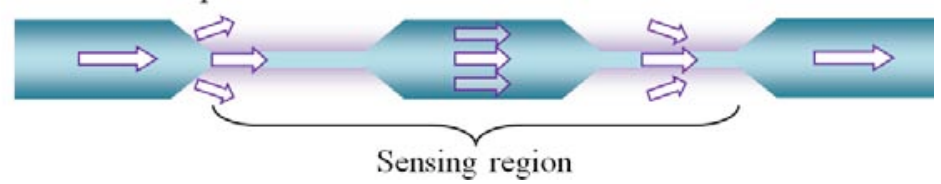

Abrupt-MNF based Michelson interferometer

(g)

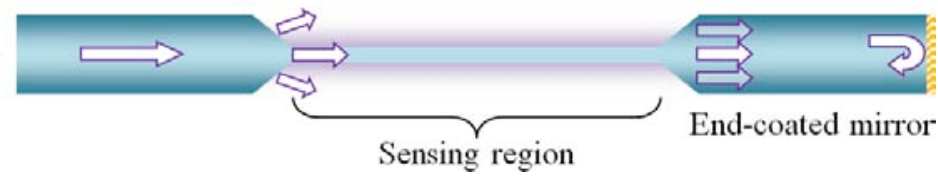

MNF tip

(h)

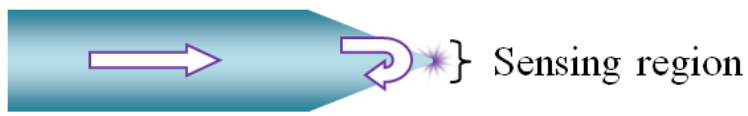

Uncoupled MNF coil

(i)

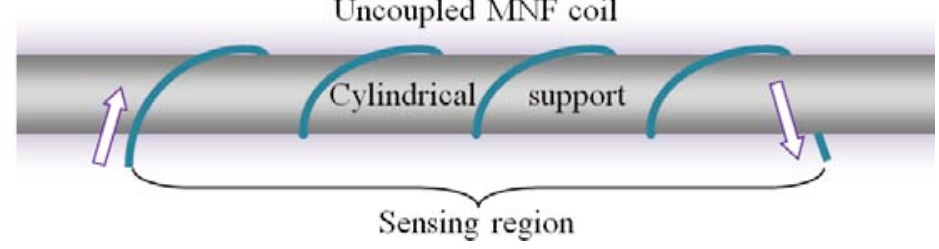

Fig. (3). Illustration of common non-resonator-type MNF structures reported in literature.

hollow MNFs have also been proposed [14]. Large evanescent field is particular important in sensors and resonators, where a significant fraction of the propagating power needs to interact with surrounding medium.

\subsection{Bend Insensitivity}

MNFs have excellent mechanical properties which allow them to be bent and manipulated without physical damage. Due to the large index contrast between silica and air [15], bending radii of the order of a few microns can be readily achieved with relatively low bend-induced loss $(<1 \mathrm{~dB}$ for 90 turn with a bending radius of $5 \mu \mathrm{m}$ in an air-clad 530 $\mathrm{nm}$ diameter silica wire [16]). This gives rise to highly com- pact devices with complex geometry, e.g. two-dimension (2D) and three-dimension (3D) multi-ring resonators.

\section{SENSOR PARAMETERS}

The performance of sensors is typically governed by a set of parameters including: sensitivity, resolution, detection limit, response time, operating range, repeatability and reproducibility.

\subsection{Sensitivity}

Sensitivity describes the change in the detected parameter as a result of a change in the measurand. For example, in the case of refractometric sensors a change in the refractive in- 
dex (measured in refractive index units - RIU) is very often understood in terms of wavelength shift relative to an initial wavelength. This detection parameter is measured in $\mathrm{nm}$, yielding the value of sensitivity in $\mathrm{nm} / \mathrm{RIU}$.

\subsection{Resolution of Detection System}

Resolution indicates the smallest detectable change in the parameter used for detection. The resolution is related to the precision with which the measurement is made and hence is usually affected by the specifications of the detection system.

\subsection{Detection Limit (DL) of Measurand}

Detection limit (DL) represents the smallest value of the measurand that can be detected by the sensor. It is related to the resolution and sensitivity by the following relationship:

$$
\mathrm{DL}=\frac{\text { Resolution }}{\text { Sensitivity }}
$$

\subsection{Response Time}

Response time $(\tau)$ is typically defined as the time required taken for the detection parameter to rise from $10 \%$ to $90 \%$ of its final value.

\subsection{Operating Range}

Operating range $(\mathrm{OR})$ is the range of values of the parameter under examination, which can be measured by the sensor, and hence by definition large OR values are highly desirable.

\subsection{Repeatability}

Repeatability is an indication of the agreement between the measured performances of the same sample taken under the same experimental conditions at different times. A good repeatability reflects the stability and life-time of the sensor head.

\subsection{Reproducibility}

Reproducibility is based on the agreement between the measured performances between different samples taken under the same experimental conditions. A good reproducibility is ideal for the manufacturing of multiple sensor heads with the same specifications.

\section{MICROFIBER AND NANOFIBER SENSORS}

This section summarizes all MNF based sensors reported to date for the measurement of refractive index, biochemical, temperature, current, displacement, bend, surface, acceleration, force, rotation, acoustic, electric field and magnetic field.

Non-resonator-type MNF sensors come in many different forms, as illustrated by Fig. (3). The straight MNF is a common configuration that exploits the strong evanescent field of the guided mode(s) to interact with the surrounding medium (Fig 3a) [17]. The measured effect is usually a change in either the intensity or phase of the transmitted light. The straight MNF is either connected to standard fiber pigtails or evanescently coupled to single-ended fiber tapers (Fig. 3b). To enhance the sensitivity to certain measurands, the surface of the MNF can be functionalised (Fig. 3c) to identify specific chemical or biological species [18]. Fiber Bragg gratings (FBG) [19] or long period gratings (LPG) [20] can be inscribed on the straight MNF that undergo a spectral shift in response to a change in the ambient conditions (Fig. 3d). Two strands of MNF can be manipulated to form a MachZehnder interferometer (MZI) [21] that features high sensitivity to the differential phase between the sensing and reference arms (Fig. 3e). Alternatively, abrupt tapered sections can be exploited for modal interferometry in the configuration of either MZI [22] (Fig. 3f) or Michelson interferometer (MI) [23] (Fig. 3g). Modal interferometry can also be realized with LPG on a single MNF [24], and polarimetric interferometry is possible with rectangular MNFs [25]. The MNF tip is widely established sensor head for probing and manipulating atoms and molecules [26] (Fig. 3h). The subwavelength cross-section of the tip facilitates a minimally invasive analysis of extremely small areas. MNF in an uncoupled helical arrangement can be employed for detecting rotations in the state of polarization induced by variations in the local magnetic field [27] (Fig. 3i).

Resonator-type MNF sensors comprises all sensors which exploit resonant structures. MNFs have been used to manufacture homogeneous resonant sensors, such as loop, knot and coil resonant sensors. Coiling an MNF onto itself allows modes propagating in adjacent turns to evanescently overlap and couple, thereby creating compact, high-Q resonators with predicted Q-factors as high as $10^{9}$. These resonators have many advantages including small-size, low fabrication cost and robustness, that have attracted much interest to develop them as optical sensors. A variety of configurations has been reported in the literature, and will be described in the following paragraph.

The microfiber loop resonator (MLR) is the simplest form of the homogeneous (all-MNF) micro-resonators. Fabrication begins with manufacturing the MNF and bending it into a self-coupling loop [28]. For example, translation and/or rotation stages can be used to adjust the loop to the desired size (Fig. (4a). The resulting MLRs show strong selfcoupling due to the close proximity of the waveguide with itself at the coupling region, with Q-factors of the order of $10^{5}$. The geometric shape of the MLR is maintained by electrostatic and van der Waals interactions at the point of coupling, which means it suffers from a limited stability dependent on environmental conditions [29, 30]. Embedding the MLR in polymer has been the preferred solution to provide long term-stability, although it can considerably modify the transmission spectrum [31]. Fusing the loop contact points with a $\mathrm{CO}_{2}$ laser $[32,33]$ has been proposed. Although this technique has generally negative effects relating to the Q-factor, resonators with $\mathrm{Q}>10^{5}$ have nonetheless been demonstrated [33]. Similarly, $\mathrm{CO}_{2}$ lasers have also been deployed to splice together different silica [34] and soft glass [35] MNFs. An alternative approach to increase the MLR long-term stability relies on the use of a copper support rod to preserve the MLR geometry [36]. Critical coupling has been demonstrated by tuning the resonator with thermal effects induced by current flowing in the conductor rod, 

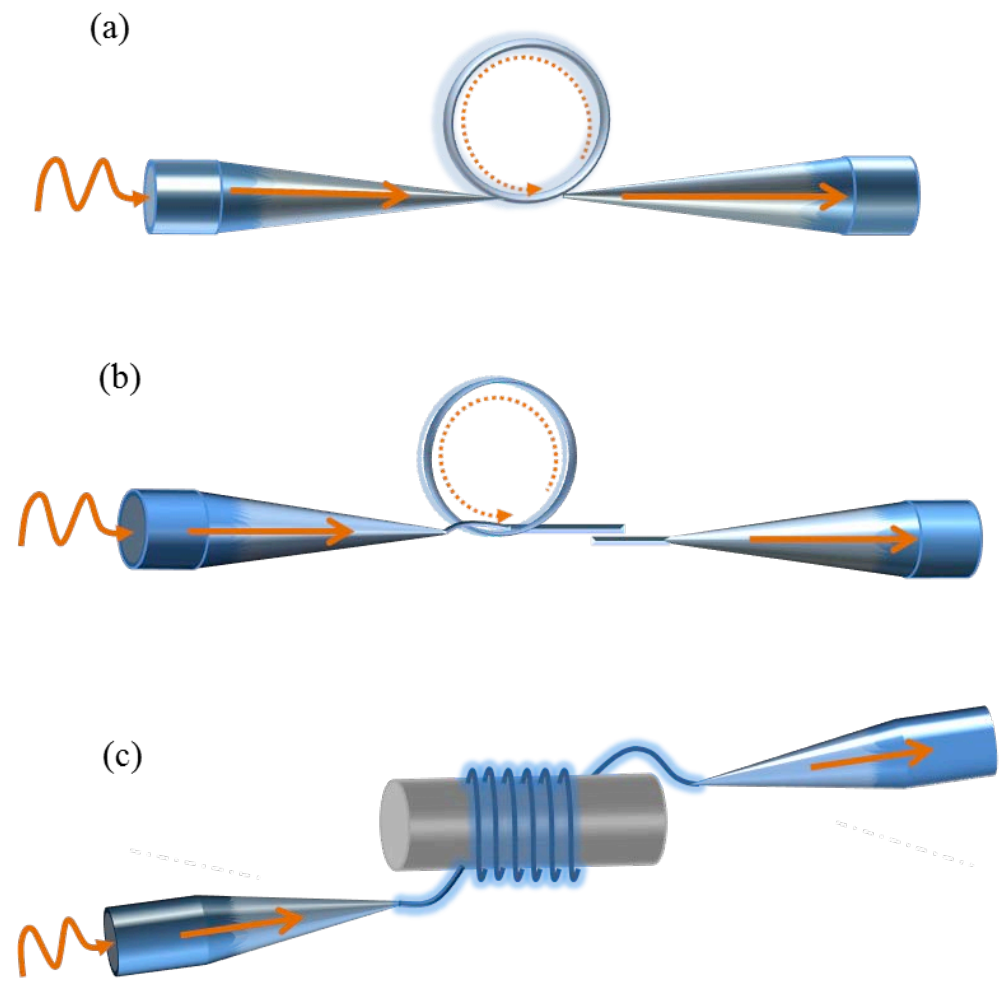

Fig. (4). Schematic of MNF homogeneous (a) loop resonator, (b) knot resonator, and (c) coil resonator. In all structures the evanescent field couples power between different sections of the same MNF.

achieving a Q-factor up to 4000 and an extinction ratio of 30 $\mathrm{dB}[37]$.

The microfiber knot resonator (MKR) can be fabricated by forming a simple knot to couple adjacent MNF sections (Fig. (4b)). This design requires less alignment precision than the MLR and benefits from improved stability: Qfactors of up to $\sim 57000$ and finesses of 22 have been achieved [38]. Q-factor and spectral properties can be tuned by changing the knot radius and tightness [39].

The micro-coil resonator (MCR) is a 3D resonator (Fig. (4c) consisting of self-coupled adjacent loops in a helix arrangement [40]. The theoretical Q-factor is in the order of $10^{9}$, competing with those achieved using whispering gallery mode (WGM) resonators [41-43]. MCRs were first experimentally demonstrated in 2007 [44], and subsequently implemented in various applications [45-51]. The highest Qfactor experimentally achieved in an MCR, at $Q \sim 470,000$ [52], is still a few orders of magnitude smaller that the theoretical maximum. Fabrication involves coiling a $\mathrm{MNF}$ around a support rod of $\mathrm{mm} / \mathrm{cm}$ scale diameter using a linear stage that controls the pitch between the turns of the coil and a rotation stage to adjust the rotation angle of the MNF. Postfabrication, the resonator can then be embedded to improve stability by preventing the ingress of moisture and dust. The multi-turn MCR transmission has been analyzed during fabrication and additional eigenmodes present in MCRs with multiple loops were detected [53].

A large number of resonant sensors utilize a single MNF to excite or collect light from high $\mathrm{Q}$ resonators such as microspheres [54, 55], micro-toroids [56, 57], micro-capillaries $[58,59]$ or bottle resonators $[60,61]$, which are classified as heterogeneous resonating sensors. By matching the propagation constants of the mode in the MNF and the microresonator, coupling efficiencies in excess of $90 \%$ have been demonstrated [62]. Evanescent sensing in these types of high-Q resonators has been used to monitor chemical and biological elements positioned in proximity of the resonator surface.

\subsection{Refractometric Sensors}

Refractive index (RI) sensing is a prominent subject of optical sensing that has a broad range of uses including the inspection of solute concentration in aqueous solutions and quality control in the monitoring of food engineering processes. The compact form of MNF based optical sensors makes sensing RI in microfluidic channels and humidity environments highly feasible and robust.

\subsubsection{Micro-Fluidic Sensors}

The first experiments with straight MNFs to confirm its applicability for RI sensing began as early as 1986, when Lacroix et al. [17] studied the transmitted core-mode power as a function of external RI with varying taper lengths. In 2005, Polynkin et al. [63] reported a simple MNF based RI sensor consisting of a submicron-diameter optical fiber taper immersed in a transparent curable soft polymer. Liquid analyte surrounded the $3 \mathrm{~cm}$ length MNF waist, making the optical loss sensitive to the RI difference between the polymer and the liquid. The DL was estimated to be $5 \times 10^{-4}$ RIU. Kude et al. [64] demonstrated another way to fabricate MNFs for RI sensing through chemical-etching. The lengths of MNF used ranged from $0.5 \mathrm{~cm}$ to $1 \mathrm{~cm}$, and uniform waist diameters from $6 \mu \mathrm{m}$ to $18 \mu \mathrm{m}$. Sui et al. [65] showed an 
alternative method for monitoring the RI-dependent optical transmission by measuring the phase change via a differential frequency technique. Their setup has shown a minimum detectable RI variation of $\sim 10^{-5}$ for $n=1.3322-1.334$. Recently, a high sensitivity RI sensor based on a tapered multimode fiber, rather than the conventional single-mode fiber, was demonstrated by Wang et al. [66]. The MNF diameter and length were $30 \mu \mathrm{m}$ and $675 \mu \mathrm{m}$ respectively. The device was able to achieve a sensitivity of at least $1900 \mathrm{~nm} / \mathrm{RIU}$ and DL of $5.23 \times 10^{-6}$ RIU within the RI range of $n=1.33-1.44$. Biazoli et al. [67] also tapered down a multimode fiber to 55 $\mu \mathrm{m}$ spliced between two sections of single-mode fiber to produce a sensitivity of $2946 \mathrm{~nm} / \mathrm{RIU}$ in the RI range of $n=$ 1.42-1.43.

Miniaturised refractometric sensors based on tapered photonic crystal fibers (PCF) have shown higher sensitivity than regular PCFs. Qiu et al. [68] investigated, both theoretically and experimentally, the RI sensing properties of PCFs with different diameters, achieved by acid-etching instead of tapering. The maximum sensitivity obtained was 750 $\mathrm{nm} / \mathrm{RIU}, 5$ times higher than that of the un-etched version. An enhancement factor of up to 100 times can be expected by optimizing the etching process to achieve thinner and more uniform PCF-MNFs. A similar design was proposed and demonstrated by Liu et al. [69], which also used acidetching to "taper" a section of PCF spliced between two single-mode fibers. The measured sensitivity of a $60 \mu \mathrm{m}$ diameter PCF/MNF was an order of magnitude higher than that of the un-tapered version, at $500 \mathrm{~nm} / \mathrm{RIU}$. Li et al. [70] fabricated a tapered PCF modal interferometer of $30 \mu \mathrm{m}$ diameter and $2 \mathrm{~mm}$ length. The miniature PCF exhibited a sensitivity of more than $1600 \mathrm{~nm} / \mathrm{RIU}$, which is nearly 8 times as high as that of an un-tapered PCF interferometer.

One of the first metal-clad MNFs for RI sensing based on the concept of exciting surface plasmon modes was introduced by Díez et al. [18]. A thin layer of gold was evaporated on a MNF of $30 \mu \mathrm{m}$ diameter and $40 \mathrm{~mm}$ length, with the different surface plasmon modes excited by the fiber modes. A sharp optical loss occurs when the propagation constant of the guided mode matches with that of a surface plasmon mode. Hence, surface plasmon waves are strongly dependent on the RI surrounding the surface. Bueno et al. [28] have shown that by adding a dielectric layer to form an asymmetric double-layer deposition (i.e. $60 \mathrm{~nm}$ thick titanium oxide on $8 \mathrm{~nm}$ thick aluminium), the response of the device can be tuned to obtain surface plasmon mode resonances at shorter wavelengths and at a lower RI range. Monzón-Hernández et al. [71] have studied different hybrid surface plasmon modes that are excited and supported by a semi-cylindrical metallic shell (25 $\mathrm{nm}$ thick gold layer) around the MNF (27.5 $\mu \mathrm{m}$ diameter, $4 \mathrm{~mm}$ length). The transmission spectrum of the device exhibits multiple resonance peaks, three of which are simultaneously monitored. The measured sensitivity was remarkably high at 127500 $\mathrm{nm} / \mathrm{RIU}$ for RI between $n=1.44-1.454$. A DL of $7 \times 10^{-7} \mathrm{RIU}$ can be obtained, assuming a wavelength resolution of $10 \mathrm{pm}$. A tapered Bragg fiber was first proposed and demonstrated by Ma et al. [72]. A MNF of $4 \mu \mathrm{m}$ diameter and $10 \mathrm{~mm}$ length was fabricated. In their design, the propagation constant of the transmitted light can be manipulated through altering the core size in order to obtain a desired RI sensing

\section{Cross-section of support rod}

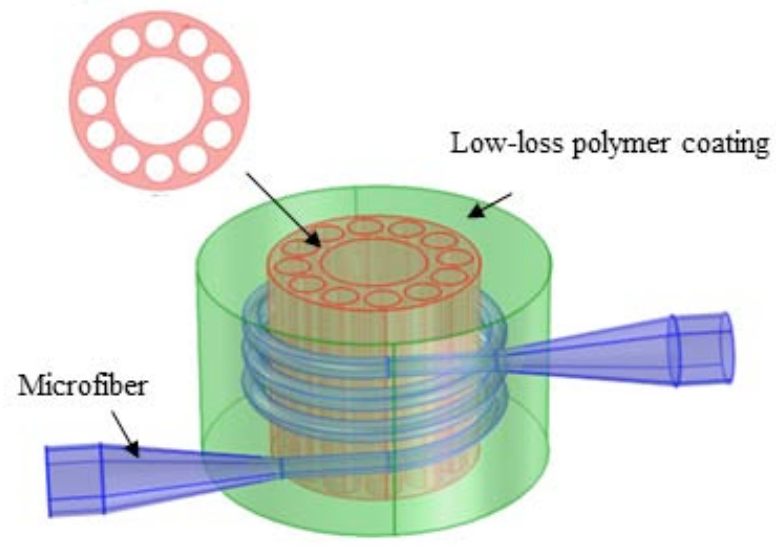

Fig. (5). Illustration of the MNF grating based on a microstructured rod. Inset: cross-section of the support rod.

range. In addition, the intensity of the interacting evanescent field can be adjusted by changing the number of Bragg multilayers. The effect of gold nanoparticles coated on a $1 \mu \mathrm{m}$ MNF was investigated by Cui et al. [73]. It was found that the resonant wavelength of the surface plasmon was determined by the size of the gold nanoparticles. By tracking the induced wavelength shift, an average sensitivity of 218.67 $\mathrm{nm}$ RIU was achieved for RI ranging from $n=1-1.362$ with particle sizes between $20 \mathrm{~nm}$ and $100 \mathrm{~nm}$. Liu et al. [74] showed that the sensitivity of a bare MNF $(2.8 \mu \mathrm{m}$ diameter, $14 \mathrm{~mm}$ length) based RI sensor can be enhanced by a factor of 100 using silica nano-spheres (400 $\mathrm{nm}$ diameter) as a modified surface to support Mie-scattering. A tapered fiber localized surface plasmon resonance sensor was demonstrated by Lin et al. [75] for RI sensing and label-free biochemical detection. The sensing mechanism is based on the evanescent field absorption of immobilized gold nanoparticles on the tapered fiber surface. A DL of $3.2 \times 10^{-5}$ RIU was reported for a MNF of $48 \mu \mathrm{m}$ diameter and $1.25 \mathrm{~mm}$ length with gold nanoparticles of $24 \mathrm{~nm}$ in average diameter.

The combination of FBGs and MNFs was first reported by Liang et al. [19]. A single etched MNF (6 $\mu \mathrm{m}$ diameter) with FBG and another MNF $(3 \mu \mathrm{m})$ with a pair of FBGs forming a Fabry-Perot interferometer was compared. In both cases, the resonant wavelength shift is proportional to the external RI. Due to the narrower resonance spectral shape of the latter configuration, a DL of $1.4 \times 10^{-5} \mathrm{RIU}$ was attained. FBGs in MNFs made with femtosecond infra-red irradiation by Grobnic et al. [76] were able to measure RI changes down to $2.5 \times 10^{-5}$. The $20 \mathrm{~mm}$ length MNFs were of $30 \mu \mathrm{m}$ and $50 \mu \mathrm{m}$ in diameter. Similarly, a $2 \mu \mathrm{m}$ diameter MNF with a sensitivity of $231.4 \mathrm{~nm} / \mathrm{RIU}$ at $n=1.44$ was shown by Fang et al. [77]. A multimode fiber taper with dual FBGs has been fabricated by Shao et al. [78], which relies on the intensity difference of two reflected signals to deduce the change of the external RI. The gratings were fabricated using a $\mathrm{KrF}$ excimer laser with a phase-mask technique. The diameter of the MNF samples was in the order of 20-30 $\mu \mathrm{m}$. The pair of FBGs makes it possible to simultaneously measure ambient temperature as a reference. The DL was approximately $\sim 10^{-5}$ for an RI range of $n=1.3333-1.4206$. Xu et al. [79] proposed a method of manufacturing gratings by wrapping a MNF around a micro-structured rod, as shown in Fig. (5). By 
exploiting the large evanescent field of MNFs, the holes in the rod can be exploited as microfluidic channels for RI sensing. This method avoids post-processing thin MNFs and enables design flexibility. By designing the air hole size and position in the micro-structured rod, index chirping can also be realized. The expected sensitivity of the device was simulated to be $10^{3} \mathrm{~nm} / \mathrm{RIU}$. Zhang et al. [80] have utilized the larger evanescent field of high-order modes to achieve a sensitivity of $102 \mathrm{~nm} / \mathrm{RIU}$ at $n=1.378$ for a $6 \mu \mathrm{m}$ diameter MNF. They claim the RI sensitivity can be further increased by decreasing the MNF diameter. More recently, Ran et al. [81] inscribed FBGs on MNFs with diameters ranging from $3.3 \mu \mathrm{m}$ to tens of $\mu \mathrm{m}$ with a $193 \mathrm{~nm}$ ArF excimer laser. They confirmed that thinner MNF and high-order modes resulted in higher RI sensitivity. The $\mathrm{LP}_{21}$ peak exhibited a sensitivity of $\sim 600 \mathrm{~nm} / \mathrm{RIU}$ at $n=1.42$.

Wang et al. [82] modeled a MNF where the external RI affects only the central segment of the grating. Due to the stop-band degeneracy and rapid emergence of spectral modes when an effective phase shift occurs, a highly sensitive sensor can be realized. A DL of $2.5 \times 10^{-6}$ RIU was estimated for this design, which is an order of magnitude better than non-phase-shifted FBGs. FBGs with high index contrast were fabricated by Liu et al. [83], using the focused ion beam milling technique. Periodic $100 \mathrm{~nm}$ deep grooves were etched on the surface of MNFs with diameters less than 2 $\mu \mathrm{m}$. Due to the high index contrast of the gratings structure, the length of the gratings-written MNF can be decreased to $\sim 500 \mu \mathrm{m}$. The sensitivity was measured to be $660 \mathrm{~nm} / \mathrm{RIU}$. The performance of FBG based MNFs as RI sensors was studied by Liang et al. [84]. Attributes such as the MNF radius, grating period and temperature cross-sensitivity were investigated theoretically. It was predicted that a $400 \mathrm{~nm}$ radius MNF FBG sensor has a maximum linear response of $1200 \mathrm{~nm} / \mathrm{RIU}$ in the RI range of $n=1.3-1.39$. Moreover, a DL of $8.3 \times 10^{-6}$ RIU can be achieved using their design. For devices featuring only a single FBG, it is difficult to separate the contributions from external RI and temperature. Recently, Ran et al. [85] have come up with a solution involving FBGs on rectangular MNFs. The grating has two Bragg peaks due to the high linear birefringence of the MNF. They exhibit identical temperature sensitivities because most of the mode is confined in the MNF and the thermal-optic effect of silica dominates the temperature response. As a result, temperature-independent RI sensing can be accomplished by monitoring the wavelength separation between the two peaks. The fabrication and characterization of a LPG based MNF RI sensor has been reported by Allsop et al. [20]. The sensor response comes in the form of a spectral feature that splits and separates with increasing external RI. A series of gratings were inscribed on a $25 \mu \mathrm{m}$ diameter MNF using a frequency-doubled argon ion laser with a point-by-point writing technique. The grating periods varied from $250-500 \mu \mathrm{m}$ and the length of all grating samples was 5 $\mathrm{cm}$. The resulting devices yielded a limiting DL of $8.5 \times 10^{-5}$ RIU in the RI range of $n=1.33-1.335$. A Fabry-Perot interferometer comprising of a length of MNF as its cavity and two FBGs as reflectors was demonstrated by Zhang et al [86]. A heterodyne technique using a dual-wavelength fiber laser was used to achieve a sensitivity of $911 \mathrm{MHz} / \mathrm{RIU}$ between $n=1.334-1.400$ with a $1.455 \mu \mathrm{m}$ diameter MNF.
It is possible to employ a pair of LPGs with a tapered section in between to form an in-line MZI. Ding et al. [24] presented such a design that operates by partially coupling light to and from a cladding-mode providing interference with the core-guided mode. A $\mathrm{KrF}$ excimer laser with a point-by-point grating writing technique was used to fabricate gratings with a period of $414 \mu \mathrm{m}$, in order to excite a cladding-mode of the ninth order for high sensitivity. A DL of $\sim 5.8 \times 10^{-6}$ can be achieved assuming the measurement system has a spectral resolution of $1 \mathrm{pm}$. The same design principles were adopted by Cárdenas-Sevilla et al. [87]. MNFs with different diameters inserted between the longperiod gratings pair were fabricated and tested. A minimum DL of $\sim 2.3 \times 10^{-4}$ RIU in a RI range of $n=1.36-1.402$ was achieved. Allsop et al. [88] provided an alternative design using only single tapered LPG (400 $\mu$ m period, $5 \mathrm{~cm}$ length) to avoid the common problem of requiring the two LPGs to be identical and $3 \mathrm{~dB}$ in strength for maximum fringe visibility. The light in this case is no longer guided by the corecladding interface but instead it is initially guided between the cladding and the external medium due to the core-mode cut-off condition along the $6 \mathrm{~mm}$ length MNF. A DL of $1 \times 10^{-4}$ RIU was obtained within a RI range of $n=1.3-1.34$. A classic Mach-Zehnder interferometer assembled using MNFs was modeled by Wu et al. [89]. Due to the large evanescent field of the MNF, a small change in the external RI can result in a large phase shift of the guided mode. Simulations using a MNF of $750 \mathrm{~nm}$ diameter and $1 \mathrm{~mm}$ length foresee the capability of detecting RI variations in the order of $10^{-6}$. An experimental demonstration was provided by Wo et al. [21], reporting sensitivity as high as $7159 \mu \mathrm{m} / \mathrm{RIU}$ with a MNF diameter of $2 \mu \mathrm{m}$. A compact MNF based modal interferometer was proposed and demonstrated by SalcedaDelgado et al. [90]. An abrupt taper of $10 \mu \mathrm{m}$ diameter and $30 \mathrm{~mm}$ uniform waist length was created with such geometry that allowed the fundamental mode to excite a high-order mode during the down-taper. The modes recombine interferometrically when they enter the up-taper transition. The minimum DL was $\sim 3.7 \times 10^{-6}$ in the RI range of $n=1.33$ 1.428. As with all interferometers, the length of the sensing region influences the sensitivity. Taghipour et al. [91] showed that by modifying a MNF LPG with gold nanoparticles, the enhanced evanescent wave and penetration depth around the sensor produces an improvement of 10.3 and 6.6 times in the wavelength and intensity sensitivities respectively. Tan et al. [92] fabricated LPGs with a pulsed $\mathrm{CO}_{2}$ laser by creating six periodical deformations along fiber length with only one scanning cycle. The length of the resulting MZI was $8.84 \mathrm{~mm}$ for a $9.5 \mu \mathrm{m}$ diameter MNF. The LPG MZI exhibits sensitivities of $2225 \mathrm{~nm} / \mathrm{RIU}$ and 11.7 $\mathrm{pm} /{ }^{\circ} \mathrm{C}$.

An in-line MNF based Michelson interferometer was first reported by Tian et al. [23], using a $500 \mathrm{~nm}$ thick gold coated end facet to reflect the core-mode and the abrupttaper-induced cladding-mode that beats together at the tapered section. The MNF has a diameter of $40 \mu \mathrm{m}$ and uniform waist length of $734 \mu \mathrm{m}$. A change in external RI produces a phase difference between the two paths of the modal interferometer that results in a wavelength shift of the interference fringes. The minimum detectable RI variation was $5.1 \times 10^{-4}$. Tian et al. [22] also created an in-line Mach- 
Zehnder modal interferometer by concatenating two MNFs together. This design utilizes one abrupt taper to couple light from the core into the cladding, and a closely followed second abrupt taper to couple the cladding-mode back to the core. The pairs of MNFs were of $40 \mu \mathrm{m}$ diameter and 707 $\mu \mathrm{m}$ length. The sensitivity to RI changes was measured to be $17.1 \mathrm{~nm} / \mathrm{RIU}$. Another double-taper based MNF RI interferometric sensor was studied by Li et al. [93]. A pair of MNFs with $56 \mu \mathrm{m}$ diameter and $18 \mathrm{~mm}$ length was created by electric-arc discharge. A high sensitivity of $580 \mathrm{~nm} / \mathrm{RIU}$ was reported, for a RI range of $n=1.3348-1.3558$. An even higher performance of $2210.84 \mathrm{~nm} / \mathrm{RIU}$ was achieved by Yang et al. [94], using similar operating principles. The difference is the creation of two abrupt tapered sections along a length of uniformly thinned fiber. Typical fabricated samples were of 35-56 $\mu \mathrm{m}$ thinned fiber diameter, 30-49 $\mu \mathrm{m}$ taper diameter and $372 \mu \mathrm{m}$ taper length. Another double-taper based interferometric sensor was reported by Chen et al. [95], with MNF diameters of $58.5 \mu \mathrm{m}$ and $53.7 \mu \mathrm{m}$, and lengths of $2.4 \mathrm{~mm}$ and $2.2 \mathrm{~mm}$. The RI sensitivity was as high as $4000 \mathrm{~nm} / \mathrm{RIU}$ at the operating wavelength of 1.61 $\mu \mathrm{m}$ and $n=1.33$. A three-cascaded-taper structure was explored by Wu et al. [96]. The first and third MNFs are of 20 $\mu \mathrm{m}$ diameter and $6.1 \mathrm{~mm}$ length. The second MNF has a diameter of $76 \mu \mathrm{m}$ and length of $698 \mu \mathrm{m}$. The diameters of the MNFs were chosen such that the core-mode couples into the silica cladding at the first tapered section, light then couples into the external medium and couples back at the second weak-tapered section, before recombining with the coremode at the third tapered section. The sensitivity of the device was $28.6 \mathrm{~nm} / \mathrm{RIU}$ within a RI range of $n=1.332-1.389$.

Wang et al. [97] fabricated a structure consisting of a small core fiber sandwiched between two standard singlemode fibers, with 20 tapers of $90 \mu \mathrm{m}$ diameter periodically written along the small core fiber using a focused $\mathrm{CO}_{2}$ laser beam. Owing to the wavelength shift of the multimode interference, an average sensitivity of $226.6 \mathrm{~nm} / \mathrm{RIU}$ has been achieved in the RI range of $n=1.33-1.38$. Zhang et al. [98] reported a MNF based MZI constructed using a down-taper of $15 \mu \mathrm{m}$ diameter and $168 \mu \mathrm{m}$ length, followed by an uptaper of $400 \mu \mathrm{m}$ diameter and $280 \mu \mathrm{m}$ length. Experimental results showed that the RI sensitivity of $82.8 \mathrm{~nm} /$ RIU for a RI range of $n=1.333-1.3869$ is nearly 5 times higher than that of the conventional MZI based on two down-tapers, and 12 times higher than that those based on two up-tapers. In addition, the sensitivity for $n=1.3869-1.4362$ was 232.8 $\mathrm{nm} / \mathrm{RIU}$. A similar configuration was investigated by Gao et $a l$. [99] with a pair of up-tapers of $167 \mu \mathrm{m}$ diameter and 425 $\mu \mathrm{m}$ length, and a down-taper of $77 \mu \mathrm{m}$ diameter and 18.2 $\mathrm{mm}$ length in-between, achieving a RI sensitivity of 86.565 $\mathrm{nm} / \mathrm{cm} / \mathrm{RIU}$ in the RI range of $n=1.3332-1.4140$. Lu et al. [100] demonstrated a new approach to RI sensing at an air and aqueous glycerol solution interface, using an inline MNF based MZI. Due to the weak coupling between the fundamental cladding mode and high-order asymmetric cladding modes, the $20 \mu \mathrm{m}$ diameter MNF is very sensitive to the external RI at $2.476 \mathrm{dBm} / \mathrm{RIU}$. Lu et al. [101] also proposed simultaneous RI and temperature sensing using a tapered bend-resistant fiber. Different phase shifts between the inner and outer cladding modes of the fiber interferometer gave rise to shifts in the interference fringes proportional to the external RI. The RI sensitivities of the inner and outer cladding modes are $4.0403 \mathrm{rad} / \mathrm{RIU}$ and $44.823 \mathrm{rad} / \mathrm{RIU}$ respectively. To differentiate RI from temperature effects in a MNF based MZI with $65 \mu \mathrm{m}$ diameter and $525 \mu \mathrm{m}$ length, Lu et al [102] utilized both S-band and C/L-band light sources. Sensitivities as high as $26.087 \mathrm{~nm} / \mathrm{RIU}$ and $0.077 \mathrm{~nm} /{ }^{\circ} \mathrm{C}$ were achieved for the interference order of 144 . A non-adiabatic MNF modal interferometer with a large taper slope was reported by $\mathrm{Ji}$ et al. [103]. In the RI range of $n=1.3707$ 1.3769 , the average sensitivity for the $4.61 \mu \mathrm{m}$ diameter MNF was $15532.24 \mathrm{~nm} / \mathrm{RIU}$ and the maximum sensitivity achieved was $18681.82 \mathrm{~nm} / \mathrm{RIU}$. With an OSA's resolution of $10 \mathrm{pm}$, the DL for RI is $5.35 \times 10^{-7}$.

An ultra-sensitive RI sensor was demonstrated by Li et al. [25], which utilizes the polarimetric interference of a rectangular, $3.29 \mu \mathrm{m}$ diameter and $32.15 \mathrm{~mm}$ length MNF. The broadband light splits into orthogonal polarizations at the highly birefringent MNF and recombines at a coupler. The measured sensitivity was $18987 \mathrm{~nm} / \mathrm{RIU}$ around $n=$ 1.33. The high sensitivity was due not only to the RI-induced birefringence variation but also to its dispersive properties. The device can detect a RI variation as small as $5.27 \times 10^{-7}$ for a wavelength resolution of $10 \mathrm{pm}$. High linear birefringence that can be created post-fabrication inside a MNF by milling a slot to break the circular geometry was proposed and modeled by Kou et al. [104]. By inserting the fiberized slot waveguide inside a fiber loop mirror configuration, a sensitivity as high as $5 \times 10^{4} \mathrm{~nm} / \mathrm{RIU}$ was predicted. Shi et al. [105] suggested an in-line Mach-Zehnder interferometer with the sensor head formed by splicing a tapered thin-core fiber between two sections of regular single-mode fibers. A portion of the transmitted light couples into the cladding of the thin-core fiber due to a mode-diameter mismatch at the splice interface. At the tapered section $(90 \mu \mathrm{m}$ diameter, 700 $\mu \mathrm{m}$ length), the cladding-mode overlaps into the external medium. At the second splice interface, the cladding-mode re-couples into the larger core of the single-mode fiber. As a result, the cladding-mode interferes with the core-mode due to the phase difference. The sensitivity was calculated to be $0.447 \mathrm{~nm}$ for a $1 \%$ change of RI that ranges between $n$ $=1.333-1.3725$. A simple in-line Mach-Zehnder interferometer was fabricated by Yang et al. [106], by applying nonaxial pull in the tapering process by a fusion splicer to form a single "S"-like fiber taper. The axial offset was $96 \mu \mathrm{m}$, the MNF diameter was $65 \mu \mathrm{m}$ and the length was $660 \mu \mathrm{m}$. There was a high insertion loss of $10 \mathrm{~dB}$ due to micro-bending. The measured sensitivity was $1590 \mathrm{~nm} / \mathrm{RIU}$ in the RI range of $n$ $=1.409-1.425$.

Tapered optical fiber tips are simple yet effective at sensing RI variations within small detection volumes. Tai et al [107] presented a metal-free sub-wavelength tip that responds to RI changes in the external medium via an intensity modulation. The numerical aperture (NA) of the sensor head was 0.12 , obtaining a light intensity change of $8000 \%$ RIU in the RI range of $n=1.3-1.4$. A compact MNF probe based on a micro-notch cavity fabricated by focused ion beam micro-machining was reported by Kou et al. [108]. The device is a hybrid Fabry-Perot and modal interferometer due to the non-adiabatic geometry of the tapered tip. A sensitivity of $110 \mathrm{~nm} / \mathrm{RIU}$ was measured. Kou et al. [109] also developed a metal-dielectric-hybrid MNF probe with milled-gratings 


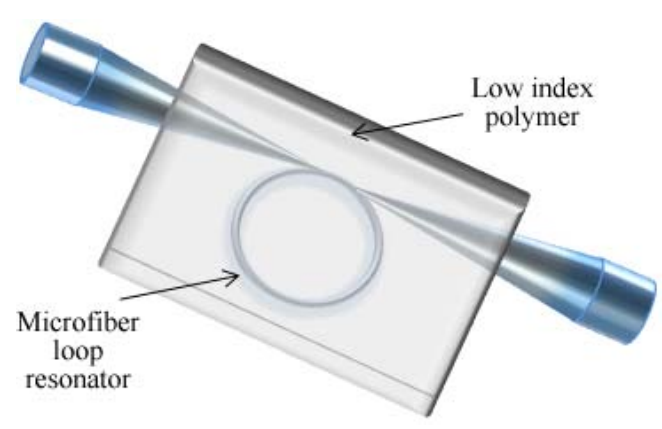

Fig. (6). Illustration of the embedded MNF loop resonator. The packaging thickness is controlled such that the evanescent field can overlap into the surrounding analyte.

replacing the micro-cavity. The $2 \mathrm{~mm}$ length tip was coated with a $30 \mathrm{~nm}$ thick layer of gold by magnetron sputtering. The gold surface provides relatively low absorption in the infrared and exhibits inertness to oxidation when exposed in air. The resulting device sensitivity was $125 \mathrm{~nm} / \mathrm{RIU}$ for RI in the range $n=1.3739-1.3577$.

A tunable refractive index sensor in the form of a $2 \times 2$ poly(trimethylene terephthalate) (PTT) MNF coupling splitter was implemented by Zhu et al. [110]. The compact structure was assembled by twisting two flexible 440-nmdiameter PTT MNFs. The sensor consisted of two input branches, a twisted coupling region and two output branches. The highest measured sensitivity was $26.96 \mathrm{~mW} / \mathrm{RIU}$ for $n=$ 1.3321-1.3565, and a minimum DL of $1.85 \times 10^{-7}$ RIU. A similar configuration was shown by Liao et al. [111], by twisting a pair of silica MNFs to form a coupling device. The highest sensitivity obtained was $2377 \mathrm{~nm} / \mathrm{RIU}$ at $n=1.368$, for the MNFs with a diameter of $4.2 \mu \mathrm{m}$. The sensitivity can be further increased to $2735 \mathrm{~nm} / \mathrm{RIU}$ for a smaller diameter of $2.8 \mu \mathrm{m}$. A hybrid device integrating a MKR in a Sagnac loop reflector was proposed for refractive index and temperature sensing by Lim et al. [112]. The fabricated MNF diameters varied from $3 \mu \mathrm{m}$ to $8 \mu \mathrm{m}$, and the sensing regions ranged from $0.5 \mathrm{~mm}$ to $2 \mathrm{~mm}$ in diameter. The RI sensitivity was $30.49 \mathrm{~nm} / \mathrm{RIU}$ in the RI range of $n=1.334-1.348$, and the temperature sensitivity was $20.6 \mathrm{pm} /{ }^{\circ} \mathrm{C}$ from $30{ }^{\circ} \mathrm{C}$ to $130{ }^{\circ} \mathrm{C}$. Layeghi et al. [113] incorporated a $300 \mu \mathrm{m}$ length of tapered high-birefringent optical fiber $(40 \mu \mathrm{m})$ into a fiber loop mirror interferometer. The resulting RI sensitivity was $389.85 \mathrm{~nm} / \mathrm{RIU}$ between $n=1.3380-1.3470$, and the temperature sensitivity was $-1.19 \mathrm{~nm} /{ }^{\circ} \mathrm{C}$ in the range of $50^{\circ} \mathrm{C}$ to $90^{\circ} \mathrm{C}$. In a Sagnac configuration, Kang et al. [114] proposed using a tapered polarization-maintaining fiber to enhance the RI sensitivity. MNFs with diameters from 36 $\mu \mathrm{m}$ to $57 \mu \mathrm{m}$ were characterized, demonstrating sensitivies from -5.59 to $-16.72 \mathrm{~nm} / \mathrm{RIU}$ in the range of $n=1.3-1.41$. Unlike the RI sensitivity dependence on the MNF diameter, their temperature sensitivities remained the same around $\sim 1.30 \mathrm{~nm} /{ }^{\circ} \mathrm{C}$. Bo et al. [115] functionalized two MNFs as a coupler with $2.5 \mu \mathrm{m}$ diameter and $2 \mathrm{~mm}$ length to measure ambient RI. Sensitivity of $2723 \mathrm{~nm} / \mathrm{RIU}$ over the RI range of $n=1.3340-1.3800$, with a maximum sensitivity of 4155 $\mathrm{nm} / \mathrm{RIU}$ between $n=1.3340-1.3515$ was reported. The DL of this device was determined to be $3.67 \times 10^{-5}$ RIU.

Resonant sensors have also been used to monitor RI. The working principle of resonant refractometric sensors is quite simple: the embedded MNF resonator has a considerable fraction of its mode propagating in the fluidic channel, thus any change in the analyte RI results in a shift in the resonant wavelength with an approximately linear dependence. Shi et al. [116] created a theoretical model for the MLR and optimized its structural parameters including the MNF radius, the loop radius and the length of coupling region for higher sensitivity, wider OR, and lower DL. Simulations indicated a DL as low as $10^{-5}$ RIU. However, as MLRs rely on electrostatic surface charges to preserve their geometry, long-term stability may become an issue in practical devices. Embedded MLRs (Fig. 6) were demonstrated by $\mathrm{Xu}$ et al. [117], and sensitivities of the order of $10^{3} \mathrm{~nm} / \mathrm{RIU}$ have been predicted for the sensor specifications of $\lambda=1.55 \mu \mathrm{m}$ and MNF radius $r \sim 300 \mathrm{~nm}$.

Guo et al. [36] reported a MLR refractometric sensor which was manufactured with the help of a copper support rod. By tuning the coupling coefficient to compensate for the circulation optical loss, critical coupling and a Q-factor of 4000 was achieved for a $2.4 \mu \mathrm{m}$ diameter MNF. This wrapped MLR has a robust structure for sensing RI in liquids and the flexibility of obtaining critical coupling within a broad spectral range. It showed high sensitivity and high stability for sensing in both low- and high- concentration solutions. By performing measurements around the wavelengths of $\lambda \sim 1.55 \mu \mathrm{m}$ and $\lambda \sim$ $1.22 \mu \mathrm{m}$, DLs of $1.1 \times 10^{-4}$ RIU and $1.8 \times 10^{-5}$ RIU were obtained in a low-concentration ethanol solution and highconcentration glycerol solution respectively. Sensitivities were $17.8 \mathrm{~nm} / \mathrm{RIU}$ and $110 \mathrm{~nm} / \mathrm{RIU}$ for high index-contrast and low index-contrast sensing respectively. Wang et al. [118] theoretically investigated MLRs for RI and salinity sensing of seawater. Dependences of sensitivity and DL on probing wavelength, MNF diameter and ring diameter were calculated. Their results showed that the sensitivity of MLRs increases with wavelength and is inversely proportional to the diameter of the MNF. By optimizing the parameters of the sensing system, RI sensitivity and salinity DL can reach $10^{-6}$ RIU and $10^{-}$ ${ }^{2} \%$ o (10 ppm) respectively.

A Mach-Zehnder interferometer coupled micro-ring was experimentally demonstrated by Wang et al. [119] that obtained a high sensitivity as well as a large OR. A very large quasi-free spectral range (>120 nm) was achieved with 400$500 \mathrm{~nm}$ diameter MNF rings. The measured sensitivity was as high as $111 \mathrm{~nm} / \mathrm{RIU}$ between a RI range of $n=1-1.538$. A miniature polarimetric interferometer consisting of a twisted highly-birefringent MNF loop was developed by Sun et al. [120]. The transmission spectral characteristics were governed by the birefringence and the twist degree of the MNF, with MNF diameters of 5.4-6 $\mu \mathrm{m}$. The structure exhibited a high sensitivity of $24373 \mathrm{~nm} / \mathrm{RIU}$ in the RI range of $n=$ 1.355-1.3586, and excellent temperature stability of better than $5 \mathrm{pm} /{ }^{\circ} \mathrm{C}$.

MKRs fabricated from multimode MNFs have also been used as refractometric sensors by Pal et al. [121]. A MKR of $<1 \mathrm{~mm}$ diameter was made from etched fibers. The highest sensitivity of $\sim 172 \mathrm{~nm} / \mathrm{RIU}$ was observed at $n=1.370$. Although MCRs have a more complex structure and they require a considerably more sophisticated fabrication procedure than other MNF resonators, they have nonetheless been explored for several sensing applications. Refractometric 
Table I. Comparison of Best-Reported Performances in each MNF Design Category for Micro-Fluidic Refractive Index Sensing

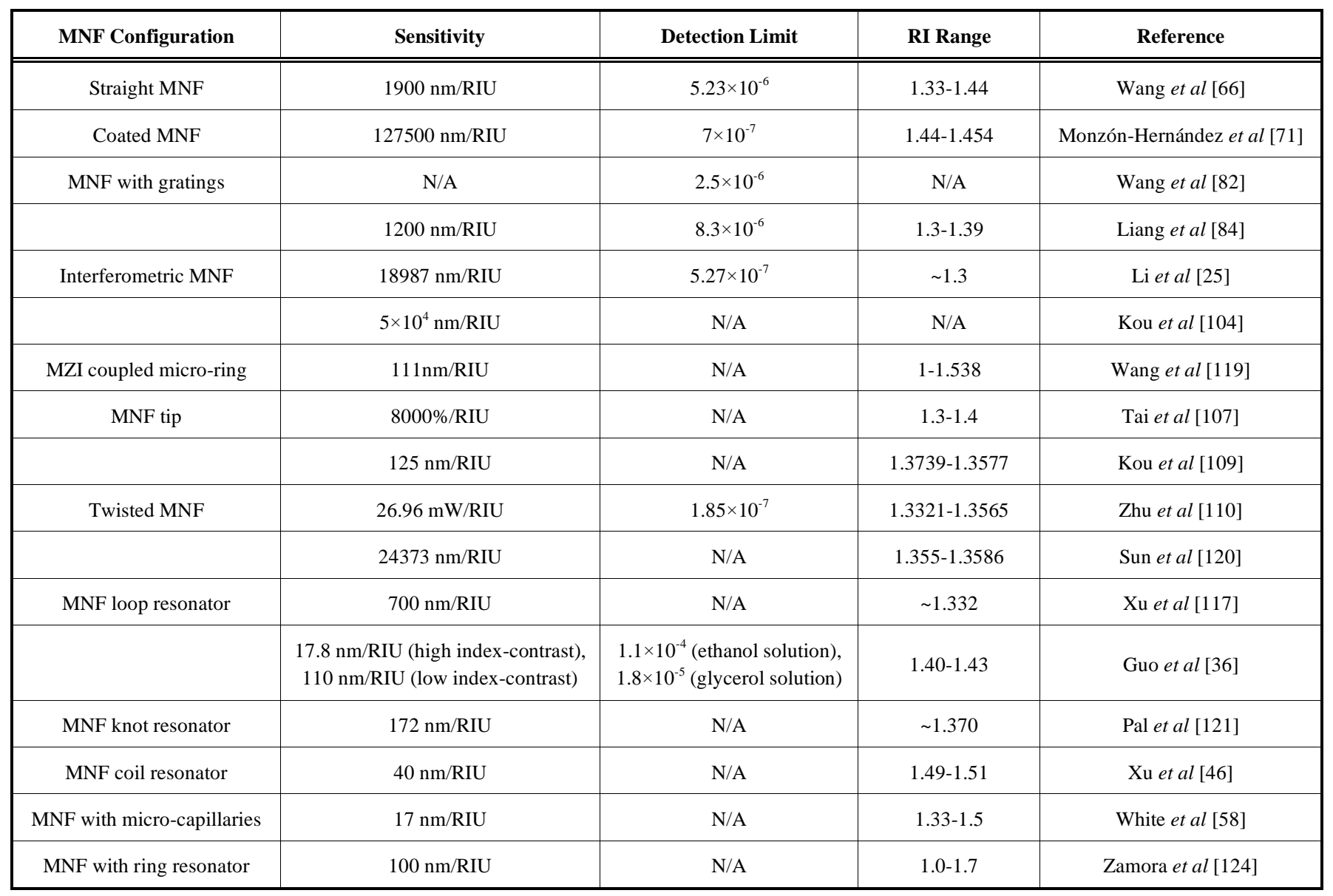

sensors based on embedded MCR have been proposed [122] and demonstrated by $\mathrm{Xu}$ et al. [46]. A MNF with $50 \mathrm{~mm}$ length and $2.5 \mu \mathrm{m}$ diameter of the uniform waist region was fabricated by the modified flame-brushing technique and then wrapped 5 times around a $1 \mathrm{~mm}$ diameter PMMA rod. The entire structure was repeatedly coated with Teflon solution to form a protective embedding layer. The dried embedded MCR was then treated with acetone to remove the support rod, which completely dissolved in 1-2 days at room temperature. Finally, an MCR sensor with a $\sim 1 \mathrm{~mm}$ diameter microfluidic channel and two input-output pigtails was obtained. Fig. (7) shows the schematic and photograph of the MCR sensor. Due to the 3D geometry, MCRs have an intrinsic channel that can be exploited for microfluidic applications. As for MLR refractometric sensors, the working principle involves the overlap between the analyte and the evanescent field of the mode propagating in the MNF. Any change in the analyte RI is reflected as a resonant wavelength shift. The wavelength shift is particularly affected by the $r / \lambda$ ratio and the coating thickness between the MNF and the fluidic channel. For optimized designs, sensitivities up to $700 \mathrm{~nm} / \mathrm{RIU}$ and DLs of the order of $10^{-7}$ have been predicted [122]. Experimental demonstration was carried out by inserting the MCR sensor in solutions of isopropanol and methanol. The resonant wavelength experienced a red shift for increasing analyte refractive indices and a sensitivity of $40 \mathrm{~nm} / \mathrm{RIU}$ was reported [46].
Micro-capillaries have been used for refractometric sensors and resonant modes in liquid core optical ringresonators and Q-factors larger than $10^{5}$ have been demonstrated by White et al [58]. The liquid flowing in the microfluidic channel has a higher RI than the surrounding polymer and the confined modes in the high-RI core are excited by an external waveguide via resonant tunnelling through the liquid core optical ring resonator. A sensitivity of $\sim 17 \mathrm{~nm} / \mathrm{RIU}$ was achieved. White et al. [123] also demonstrated an optofluidic ring resonator platform for SERS-based sensing. When silver nanoparticles and Rhodamine 6G dye were inserted in the ring-resonator, the $1360 \mathrm{~cm}^{-1}$ Raman peak was used for SERS spectroscopy, achieving a DL of $<410 \mathrm{pm}$. The sensitivity to RI changes in capillaries of submicron wall-thickness was larger than other reported optical microcavities [124]. The sensitivity was $>100 \mathrm{~nm} / \mathrm{RIU}$ for water and a maximum of $390 \mathrm{~nm} / \mathrm{RIU}$ can be achieved. Sumetsky et al. [59] demonstrated RI sensing via a polymer-matrixembedded MNF of $1.7 \mu \mathrm{m}$ diameter, coupled to a $100 \mu \mathrm{m}$ diameter capillary fiber filled with the liquid under test. A sensitivity of $800 \mathrm{~nm} / \mathrm{RIU}$ was achieved by measuring the wavelength shift of the WGMs. To summarize, a multitude of MNF-based sensors have been developed for RI sensing. Table I lists some of the most prominent achievements: 

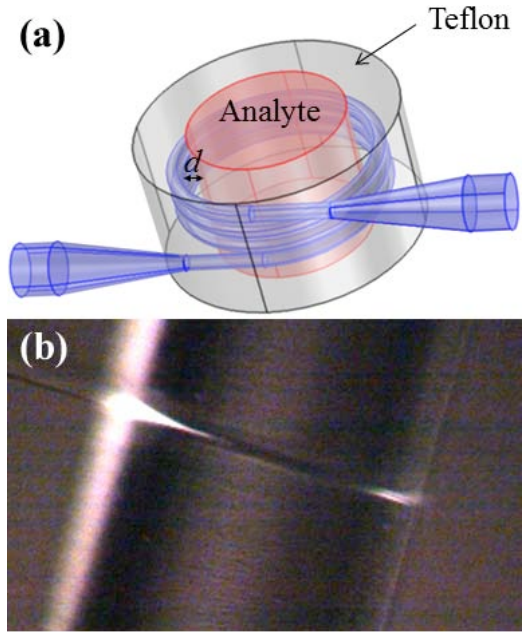

Fig. (7). (a) Illustration of the MCR sensor, and (b) photograph of the packaged sample.

\subsubsection{Humidity Sensors}

MNF based humidity sensors typically operate on RI changes in its external coating in response to variations in ambient humidity. Corres et al. [125] have coated a MNF with humidity-sensitive nano-film using poly-diallyldimethyl ammonium chloride (PDDA) and the polymeric Dye R-478 (Poly-R) as the polycation-polyanion pair that overlap each other at the molecular level to produce a homogeneous optical material. Optimization of the humidity response was studied further [126] by tuning the coating type, coating thickness, dimensions of the taper and the light source. A variation of $16 \mathrm{~dB}$ in optical transmittance was achieved with a responses time of $300 \mathrm{~ms}$ for changes in relative humidity (RH) from 75-100\% [127]. An alternative humiditysensitive material is the agarose gel, as shown by Bariáin et al. [128]. A mixture of agarose powder dissolved in water was heated and then deposited on a MNF of $25 \mu \mathrm{m}$ diameter and $0.1 \mathrm{~mm}$ uniform waist length. A variation of up to 6.5 $\mathrm{dB}$ of the optical transmittance was recorded with $\mathrm{RH}$ changes between $30 \%$ and $80 \%$. Gelatin is another material sensitive to humidity changes. Zhang et al. [129] exploited this effect by coating a $680 \mathrm{~nm}$ diameter, $8 \mathrm{~mm}$ length MNF with an $80 \mathrm{~nm}$ thick layer of gelatin. The sensor displayed a $10 \mathrm{dBm}$ change in optical transmittance with an OR of 9$94 \% \mathrm{RH}$, with $\tau=70 \mathrm{~ms}$. A fast response time of $\tau=30 \mathrm{~ms}$ was demonstrated by $\mathrm{Gu}$ et al. [9] when exposed to an at- mosphere of RH between $35-88 \%$. In their setup, a $410 \mathrm{~nm}$ diameter, $250 \mu \mathrm{m}$ long polyacrylamide MNF was supported on an $\mathrm{MgF}_{2}$ substrate and evanescently coupled to fiber tapers at both ends. Li et al. [130] proposed and demonstrated a polyvinyl-alcohol-coated multimode fiber taper cascaded with a FBG. This scheme utilizes the reflective grating to double the humidity-dependent-attenuation of the transmitted light. A maximum sensitivity of $1.994 \mu \mathrm{W} / \% \mathrm{RH}$ was reported within the OR of 30-95\% RH with a MNF diameter of $50.2 \mu \mathrm{m}$. The average $\tau$ of the system was $2 \mathrm{~s}$. More recently, Meng et al. [131] investigated a quantum-dotactivated MNF sensor (480 nm diameter) for humidity detection with a very fast response $(<90 \mathrm{~ms})$ and an extremely low power consumption.

A humidity sensor based on tapered PCF filled with moisture-sensitive polymer was proposed by Li et al. [132]. Theoretical results show the loss varies from $0.063 \mathrm{~dB} / \mathrm{cm}$ to $75.847 \mathrm{~dB} / \mathrm{cm}$ when the $\mathrm{RH}$ changes from $0 \% \mathrm{RH}$ to $95 \%$ RH. Humidity sensing using MKRs has been reported [64]. The silica MKR has showed a sensitivity of $\sim 12 \mathrm{pm} / 10 \% \mathrm{RH}$ within an OR of 14-60\% RH.

Wang et al. [133] fabricated micro-rings assembled with polyacrylamide (PAM) MNFs of 2-3 $\mu \mathrm{m}$ diameter for humidity sensing. PAM MNFs absorb water molecules and inflates monotonically with increasing humidity, resulting in resonant wavelength shifts. Sensitivities as high as 490 $\mathrm{pm} / \% \mathrm{RH}$ and response times of $\tau=120 \mathrm{~ms}$ were recorded within an OR of 5-71\% RH. Interferometric humidity sensors based on silica/polymer MKRs were reported by Wu et al. [134]. The silica MKR sensor (1.2 $\mu \mathrm{m}$ diameter, $\mathrm{Q}$ of 15000) factor showed a sensitivity of $12 \mathrm{pm} / 10 \%-\mathrm{RH}$ within an OR of $15 \%-60 \%$ RH. The polymer MKR sensor $(2.1 \mu \mathrm{m}$ diameter, $\mathrm{Q}$ of 20000) achieved a sensitivity of $88 \mathrm{pm} / 10 \%$-RH from $17 \%$ 95\% RH, with a temporal response of $\tau<0.5 \mathrm{~s}$.

\subsection{Bio-Chemical Sensors}

There has been a rapid development of optical sensors for the detection of chemicals and biological materials of environmental and biomedical interest. The emergence of MNF based devices gave rise to the capability of interro-gating samples in the microscopic size regime with minimized intrusion, greater stability and shorter response times. In the following section, sensors are categorized mainly by their sensing mechanism.

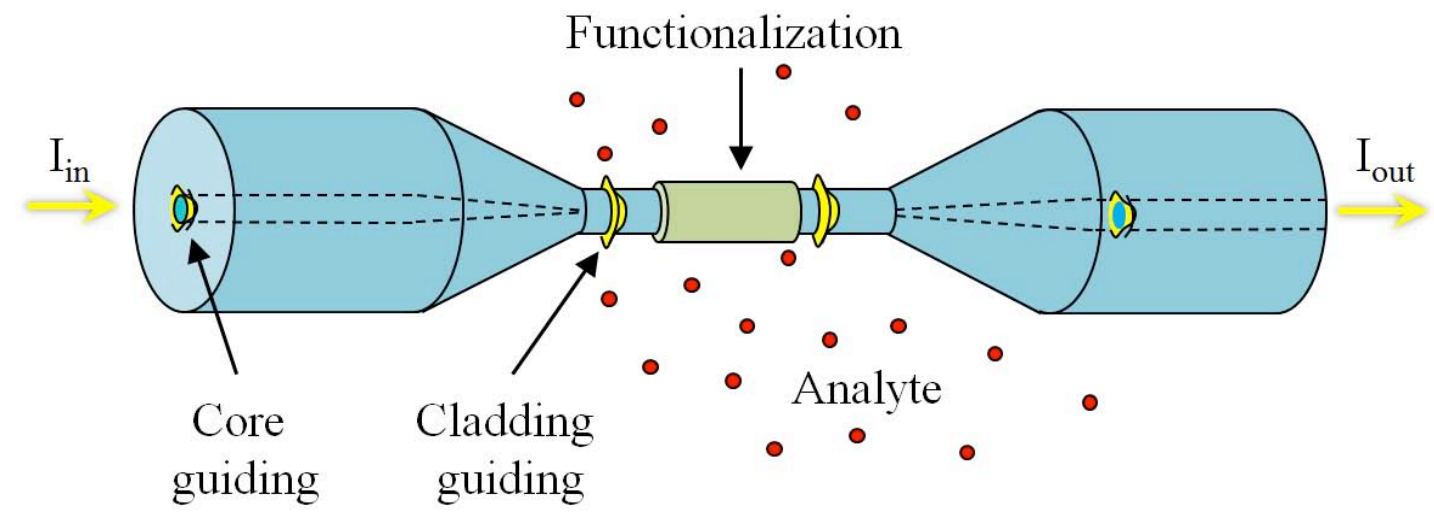

Fig. (8). Schematic diagram of a coated MNF for bio-chemical sensing. 


\subsubsection{Surface Absorption and Fluorescence Sensors}

The strong optical confinement and prominent evanescent field of guided light in MNFs give rise to ultra-sensitive surface spectroscopy of molecules in close vicinity of the MNF waist region (Fig. 8). Using the guided mode for both excitation and fluorescence collection, Stiebeiner et al. [135] performed spectroscopic measurements on 3,4,9,10perylenetetracarboxylic dianhydride molecules (PTCDA) at ambient conditions, using MNFs with waist diameter down to $100 \mathrm{~nm}$ and lengths of $1-10 \mathrm{~mm}$. Fine-tuning of the transmission band of MNFs allow for further optimization on the surface spectroscopy of organic molecules. A systematic study of the influence of the fiber profile parameters on the transmission properties of MNFs as well as the loss mechanisms was also undertaken by Stiebeiner et al. [2]. A MNF with $120 \mathrm{~nm}$ diameter resulted in a transmission of $70 \%$ at $485 \mathrm{~nm}$, with more than $99 \%$ of the optical power of the light guided outside the MNF. Warken et al. [136] performed surface absorption measurements on a MNF of 500 $\mathrm{nm}$ diameter and $3 \mathrm{~mm}$ length with sub-monolayers of 3,4,9,10-perylene-tetracarboxylic dianhydride (PTCDA) molecules at ambient conditions. It was concluded that the sensitivity exceeded free-beam surface absorption spectroscopy by several orders of magnitude. Takiguchi et al. [137] performed saturated absorption spectroscopy of acetylene $\left(\mathrm{C}_{2} \mathrm{H}_{2}\right) v 1+v 3$ band transitions with a MNF. The saturation parameter was maximized at a MNF diameter of $410 \mathrm{~nm}$. The measured homogeneous linewidth was $51 \mathrm{MHz}$, and the resolution of the MNF spectrometer was $31 \mathrm{MHz}$. Due to the high intensity evanescent field surrounding the MNF, a Lamb dip was observed using a relatively low powered laser without a cavity. A spectrophotometer based on tapered multimode fiber was developed by Vishnoi et al. [138], by removing the cladding in the central portion and tapering. Different tapering ratios of 1.5, 1.82, 2.0 and 2.4 were prepared with the tapering length of $1.2 \mathrm{~cm}$. It was found that $\tau$ decreases with increasing tapering ratio. Aqueous dye solutions of concentrations as low as $1 \mathrm{ppm}$ could be detected using their setup. Zhang et al. [139] reported a compact absorption sensor by using a $900 \mathrm{~nm}$ diameter MNF embedded in a $125 \mathrm{~mm}$ wide micro-channel with a detection length of $2.5 \mathrm{~cm}$. By measuring the absorbance of methylene blue (MB), the sensor showed a DL down to $50 \mathrm{pM}$ within the concentration OR of 0-5 nM. The sensor was also functionalised to test bovine serum albumin (BSA) and achieved a DL of $10 \mathrm{fg} \mathrm{mL}^{-1}$.

Wiejata et al. [140] fabricated MNFs with $3.69 \mu \mathrm{m}$ diameter and $7.1 \mathrm{~mm}$ length for fluorescent sensing. Flourescein solution at concentrations of $10-60 \mu \mathrm{M}$ was placed in the waist region. The MNF was excited with light at $460 \mathrm{~nm}$ and the emitted fluorescent light was collected at $516 \mathrm{~nm}$. The coupling efficiency of the spontaneous emission of atoms to a MNF was theoretically investigated by Le Kien et al. [141]. For a MNF diameter of $400 \mathrm{~nm}$, it was found that as much as $28 \%$ of the spontaneous emission by a caesium atom could be converted into guided modes along the MNF. A systematic formalism was subsequently developed [142] to calculate the correlation functions of fluorescence from a multi-atom system into guided modes of a MNF. Fiber-mediated superfluorescence was shown to be possible when the spacing of the atoms in a linear array is an integer multiple of the wavelength of the resonant guided modes. Das et al. [143] showed that the fluorescence emission spectrum of a few cold atoms can be investigated using MNFs combined with an optical heterodyne technique and photon correlation spectroscopy. A MNF of $400 \mathrm{~nm}$ diameter and $2 \mathrm{~mm}$ length was used to measure 14 laser-cooled C atoms, by integrating the fluorescence photons for $3 \mathrm{~min}$. More recently, Nayak et al. [144] discovered that the fluorescence excitation spectrum line-shape of cold atoms in the vicinity of a MNF evolve with time, due to changing surface conditions. It was experimentally shown that the loading into such surface bound-states could be controlled by maintaining the surface conditions. The label-free detection of biomolecules based on the modal interference along a nonadiabatic MNF was demonstrated by Tian et al. [145], with a micro-channel chip designed and fabricated for the sensor's protection and bio-testing of an $\operatorname{IgG}$ antibody-antigen pair. The modeling and analysis of localized bio-sensing based on an effective phase shift in FBG-inscribed MNFs was presented by Wang et al. [82]. The stop-band degeneracy and rapid emergence of spectral modes was initially exploited for RI sensing and it can also be used to detect surface protein or DNA adsorption with a DL as low as $3.3 \mathrm{pg} . \mathrm{mm}^{-2}$ for surface mass density and $51.8 \mathrm{fg}$ for total mass.

The influence of the van der Waals interaction between atoms and the surface of MNFs was studied by Minogin et al. [146]. For MNF diameters of 100-1000 nm, the van der Waals red shift was found to increase the red half-width of the spontaneous emission line by $10-30 \%$ while the blue half-width of the spectral line remain unchanged. Nayak et al [147] demonstrated efficient coupling of atomic fluorescence to the guided mode of a MNF. It was reported that the fluorescence of a very small number of atoms could be detected around MNFs with diameters of 100-1000 nm and $40 \mathrm{~mm}$ length. Likewise, they also confirmed that the fluorescence excitation spectrum reflects the effect of the van der Waals interaction between atoms and the MNF surface. Moreover, Nayak et al. [148] discovered that single atoms around the MNF reveal a very prominent spectral feature, where the excitation spectrum splits into two peaks with a separation smaller than the natural linewidth. The potential of MNFs for detecting, trapping, and manipulating cold atoms was explored by Sagué et al. [149]. The interaction of a small number of cold caesium atoms with the guided mode of a $500 \mathrm{~nm}$ diameter, $5 \mathrm{~mm}$ length MNF and the fiber surface was investigated using high-resolution spectroscopy. Light-induced dipole forces, van der Waals interaction, and a significant enhancement of the spontaneous emission rate of the atoms were observed and discussed. Russell et al. [150] analyzed the line-shape of the fluorescence emitted by a cloud of optically excited cold atoms that was evanescently coupled into a MNF. The efficiency of the fluorescence coupling was studied and the asymmetry of the line-shape was explained by the redshifts arising from both the van der Waals and Casimir-Polder interaction of the atoms with the surface of the MNF. As a result, the number of atoms and the strength of interaction can be deduced by measuring the line-shape of the coupled fluorescence. From the Rayleigh-Gans scattering theory of spherical particles and evanescent-wave guiding properties of MNFs, Wang et al. [151] carried out simulations based on the scattered power of nanoparticles in the 
vicinity of MNFs. Theoretical analysis suggest that nanoparticle-induced scattering intensity can reach a detectable level by optimizing the wavelength of the probing light and the diameter of the MNF, opening up new possibilities for single-molecule detection.

A MNF based $\mathrm{pH}$ sensor was presented by Baldini et al [152]. An acid-base indicator thymol blue (TB) was covalently immobilized on the core of a 200/380 $\mu$ m fiber with an interaction length of $8 \mathrm{~mm}$ by silylation processing the glass surface. It was found that a tapering ratio of (fiber diameter/waist diameter) of 2.3 lead to an enhancement in the evanescent-field-based sensitivity by a factor 6 , and a DL of $0.05 \mathrm{pH}$ units was attained. Lossy resonances generated by a multi-layered assembly of poly (allylamine hydrochloride) and poly(acrylic acid) on a $30 \mu \mathrm{m}$ diameter, $10 \mathrm{~mm}$ length MNF was exploited by Socorro et al. [153] as a pH sensor by detecting the wavelength shift associated with changes in $\mathrm{pH}$ level. A sensitivity of $87.5 \mathrm{~nm} / \mathrm{pH}$ unit was observed between $\mathrm{pH} 4-6$, and the DL was estimated to be $0.05 \mathrm{pH}$ units. The response time for detecting a $\mathrm{pH}$ change was recorded to be $60 \mathrm{~s}$. A MNF based surface plasmon resonance sensor for direct monitoring of bio-molecular interactions and chemical and biological reactions was developed by Monzón-Hernández et al. [154]. Multiple resonance peaks were visible when $4 \mathrm{~mm}$ length MNFs with diameters ranging from $25 \mu \mathrm{m}$ to $40 \mu \mathrm{m}$ were coated on one side with a 26 $\mathrm{nm}$ thick layer of gold. This characteristic was experimentally verified to increase the sensing dynamic range remarkably. A label-free detection of quadruplex formation of peroxidase-like DNAzyme using the RI sensing ability of MNFs was investigated by Bagheri et al. [155]. The nonadiabatic MNF used in their work is around 8-9 $\mu \mathrm{m}$ in diameter and 10-15 $\mathrm{mm}$ in length. A study of the antibacterial effect of silver nanoparticles attached to MNFs with 6-8 $\mu \mathrm{m}$ diameter and 17-20 mm length was presented by Saeedian et al. [156]. It was found that by anchoring and penetrating the bacterial cell with silver nanoparticles, the RI perturbation can lead to shifts in the optical transmission of the MNF. A highly sensitive and fast response molecular sensor based on a graphene-coated MNF was demonstrated by Yao et al. [157]. Since the complex RI of graphene can be modulated by its local boundary conditions when molecules are attached on the surface, molecular concentrations can be measured by an intensity measurement of the transverseelectric optical mode. MNFs of $1 \mu \mathrm{m}$ and $1.5 \mu \mathrm{m}$ diameters were employed as the emitting and collecting fibers, which were coupled together by van der Waals forces before being deposited by a layer of graphene by pressure spin coating.

MNF tip sensors facilitate sensitive and selective means to monitor cellular microenvironments at the single cell level. Tan et al. [26] were amongst the first to realize this vast potential. A thousand-fold probe miniaturization, a million-fold sample reduction and a hundred-fold shorter response time $(\tau<20 \mathrm{~ms}$ ) was achieved by combining MNF tips with near-field photo-polymerization. The wide variety of medical uses at the cellular level was discussed by VoDinh et al. [158]. In their report, MNF tips of 30-50 nm diameter were covalently bound with antibodies that are selective to target analyte molecules (Fig. 9). The sensor head was excited by light launched into its fiber pigtail and the resulting evanescent field at the MNF tip was used to excite target

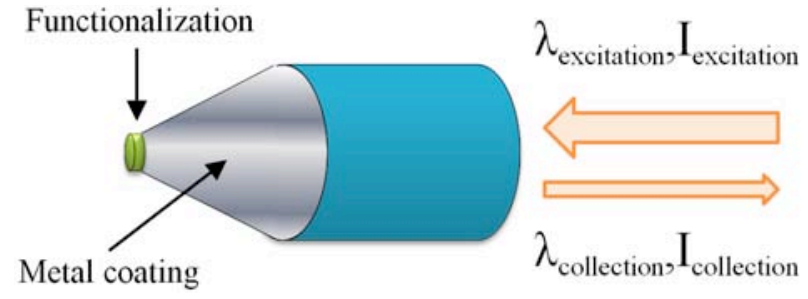

Fig. (9). Schematic diagram of a functionalized MNF tip for selective bio-chemical sensing.

molecules bound to the antibody molecules. The fluorescence emission from the analyte molecules was then collected and analyzed via a microscope. Fluorescence intensity based sensing depends on many parameters, such as light source, collection geometry, and quenching effects; therefore, quantification is usually complicated. Ratio measurements were established as a common method to quantify fluorescence signals using a sensing and a reference dye. McCulloch et al. [159] demonstrated a miniaturized $\mathrm{pH}$ micro-optrode by immobilising a fluorescent $\mathrm{pH}$-sensitive reagent within a glass film and deposited on the surface of a sub-micron diameter MNF tip using a sol-gel process. The fluorescence yield of the $\mathrm{pH}$-sensitive reagent provided an indication of the $\mathrm{pH}$ level, which ranges from 3 to 10. Optochemical sensors for $\mathrm{pH}$ and chloride were developed by Koronczi et al. [160], by coating silanised MNF tips of 300 $\mathrm{nm}$ diameter with polymeric membranes containing luminescent indicators. Luminescence decay time was measured using a phase-modulation technique. A DL of 0.1 units of $\mathrm{pH}$ or $\mathrm{pCl}$ was obtained. A pH sensitive nano-optrode based on surface-enhanced Raman scattering (SERS) detection was proposed and demonstrated by Scaffidi et al. [161], as an alternative to fluorescence-based techniques. The fabricated MNF tip of $<100 \mathrm{~nm}$ diameter was coated with a $6 \mathrm{~nm}$ thick layer of silver to make it plasmonics-active. Zamarreno et al. [162] described an improvement factor of 5 over the reduction in response time of a MNF tip coated with a $\mathrm{pH}$ sensitive film, by depositing a hydrophilic nanostructured layer before the $\mathrm{pH}$ sensitive coating. A MNF tip based detection of dissolved ammonia in concentrations below $100 \mu \mathrm{gL}^{-1}$ was presented by Waich et al. [163]. The sensing principle involves a fluorescent $\mathrm{pH}$ indicator bound to the tip of a MNF (diameters between 10-20 $\mu \mathrm{m}$ ) that is deprotonated by the presence of ammonia. A detection limit of $0.5 \mu \mathrm{g} \mathrm{L}^{-1}$ was achieved within the OR of $0.5-100 \mu \mathrm{gL}^{-1}$, under a $15 \mathrm{~min}$ response time.

Nitrite- and chloride-selective fluorescent nano-optrodes made by tapering conventional sized optical fibers down to 0.3-0.7 $\mu \mathrm{m}$ in diameter were demonstrated by Barker et al. [164]. One end of the MNF waist was aluminium-coated and either prepared with a vitamin $\mathrm{B}_{12}$ derivative ionophore to sense nitrate, or prepared with an indium porphyrin ionophore to detect chloride. For the nitrite nano-optrode, the sensing dynamic range was $5 \mu \mathrm{M}$ to $14 \mathrm{mM}$ at $\mathrm{pH}$ 7.4. The DL was measured to be $2 \mu \mathrm{M}$ activity nitrite. For the chloride nano-optrode, the sensing dynamic range was $63 \mathrm{mM}$ to $100 \mathrm{M}$ at $\mathrm{pH}$ 7.4. The limit of detection was measured to be $16 \mathrm{mM}$ activity chloride. Nitric-oxide-specific sensors of 200-500 nm tip diameter prepared based on the fluorescence of the cytochromes c [165] achieved a response time of $\tau<1$ $\mathrm{s}$. The response to nitric oxide was linear up to $1 \mathrm{mM}$, with a 


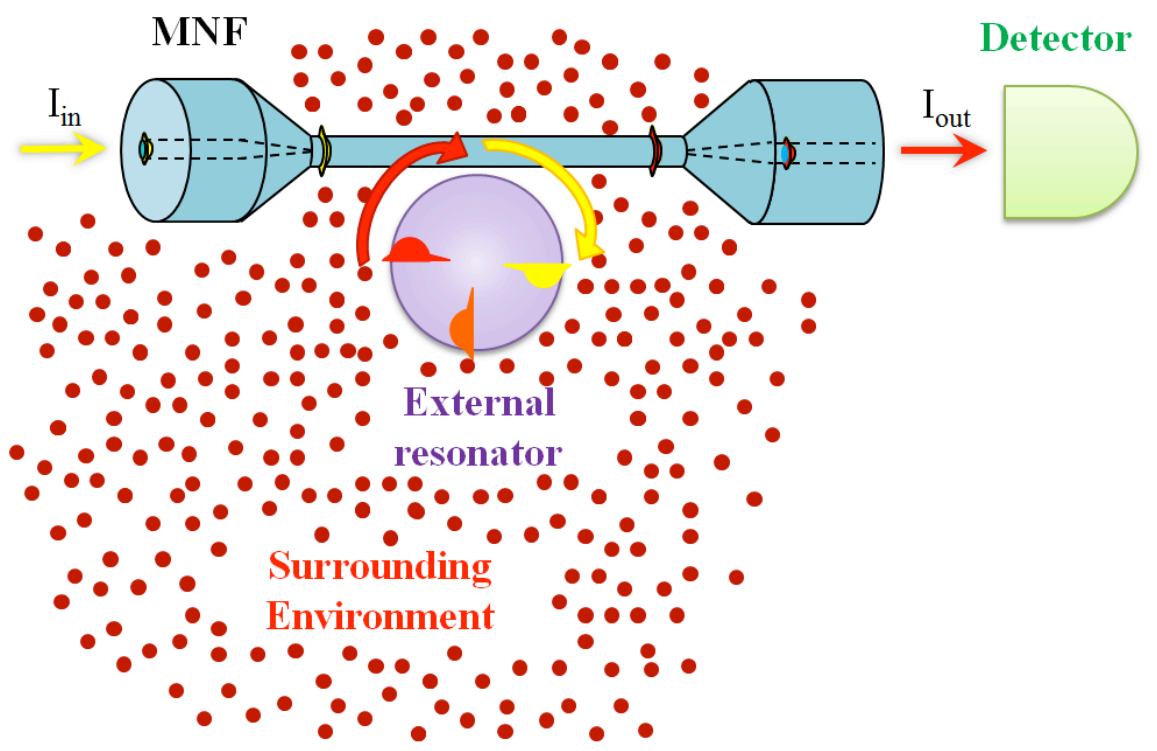

Fig. (10). Schematic diagram of a MNF coupled to a microsphere for biological detection.

DL of $20 \mu \mathrm{M}$. A different $\mathrm{NO}_{2}$ selective sensor design was presented [166], which involves placing a tapered multimode fiber in a $50 \mathrm{~nm}$ gold colloid, before being coated with a dilute suspension of fluorescent carboxylate-modified polystyrene microspheres (40 $\mathrm{nm}$ diameter) with $488 \mathrm{~nm}$ excitation and $685 \mathrm{~nm}$ emission wavelengths. Fluorescence changes in the presence of $\mathrm{NO}_{2}$ were observed to be as fast as $0.25 \mathrm{~s}$. The measurements were made ratiometric by the addition of reference dye microspheres. The intracellular measurement of cytochrome $\mathrm{c}$ itself using a MNF was presented by Song et al. [167]. Plastic-clad silica fibers with $600 \mu \mathrm{m}$ diameter core were pulled to a tip diameter of $40 \mathrm{~nm}$ using a laser-based micro-pipet pulling device, before being coated with silver to attain a final tip diameter of $150 \mathrm{~nm}$. The resulting MNF probe combined with an enzyme-linked immunosorbent assay (ELISA) facilitated high sensitivity detection due to the enzymatic amplification of target fluorescence on the MNF. A glutamate sensor was introduced by Cordek et al. [168] by covalently binding glutamate dehydrogenase to a MNF tip surface. The detection of glutamate was made possible by monitoring the fluorescence of the reaction product, nicotinamide adenine dinucleotide (NADH). The concentration DL was measured to be 0.22 $\mu \mathrm{M}$ glutamate, with $\tau<50 \mathrm{~ms}$.

The development of MNFs in bio-sensing has also lead to measurements of toxic chemicals within single cells. Cullum et al. [169] fabricated MNF tip diameters of less than $1 \mu \mathrm{m}$ and coated the end-faces with antibodies to selectively bind the species of interest, namely benzo[a]pyrene tetrol (BPT) that is a metabolite of benzo[a]pyrene. A DL of $\sim 0.64 \times 10^{-11}$ $\mathrm{M}$ was reported for BPT. Another antibody-based MNF sensor that offers in situ measurements of the concentration of carcinogen benzo[a]pyrene in a single cell was manufactured by Kasili et al. [170]. Kasili et al. [171] have also shown the capability of MNFs to detect Caspase-9 activity in a single cell. Leucine-GlutamicAcid-Histidine-AsparticAcid-7-amino-4-methylcoumarin (LEHD-AMC) was covalently attached to the tip of a MNF and cleaved during apoptosis by caspase- 9 to generate free AMC. The evanescent field of a $50 \mathrm{~nm}$ diameter MNF tip plus $100 \mathrm{~nm}$ silver coating was used to excite the cleaved AMC and collect the fluorescence signal resulting from Caspase- 9 activity. Fluorescence-based MNF biosensors have been applied in DNA/RNA detection without the need for a dye-labeled target molecule or an intercalation reagent in the testing solution, as reported by Liu et al. [172]. Experimental results have shown that the concentration detection limit and mass detection limit for a 105 $\mu \mathrm{m}$ diameter fiber tip and a sub-micron diameter MNF tip are $0.3 \mathrm{nM}$ and $15 \mathrm{aM}$, and $10 \mathrm{nM}$ and $0.27 \mathrm{aM}$ respectively. Zheng et al. [173] have demonstrated that MNF tips can be inserted into the nucleus of living MCF-7 cancer cells to detect a biomarker, telomerase. The fluorescence response indicted a successful detection of telomerase over-expression in a single living cell for the first time, making it a potential method for general cancer detection. Plaschke et al. [174] described a new immobilization concept which consists of the encapsulation of dextran-linked fluorescence indicators in an organic hydrogel. This concept allows co-immobilization and stable encapsulation of different indicators. A compact micro-ball lens structure fabricated at the cleaved tip of a MNF coupler was proposed and demonstrated by Harun et al. [175] for sensing various glucose concentrations in deionized water. The sensing mechanism of this device is based on the change in the peak power of the transmission spectrum as the concentration of glucose is altered. The MNF and micro-ball lens measure $40 \mu \mathrm{m}$ and $102 \mu \mathrm{m}$ in diameter respectively. For a concentration change from 0-12 volume $\%$, the output intensity of the sensor decreases linearly from -57.4 to $-60.5 \mathrm{dBm}$ with a sensitivity of 0.26 $\mathrm{dB} / \%$.

An in-line absorption sensor was fabricated with an embedded MCR by Lorenzi et al. [176]. The sensor was tested by measuring the optical absorption of an aqueous solution of Brilliant Blue FCF $\left(\varepsilon=1.3 \times 10^{5} \mathrm{M}^{-1} \mathrm{~cm}^{-1}\right.$ at $\left.\lambda=630 \mathrm{~nm}\right)$ with concentration ranging from $6 \mu \mathrm{M}$ up to $0.18 \mathrm{mM}$. The measurements were taken while the analyte flowed through the tube enclosed by the MCR. The experimental results have been interpreted as a function of analyte concentration. Low concentrations of flowing analyte show optical losses in agreement with a modified Beer-Lambert law and higher 
concentration reached a limit of the measured optical losses arising from absorption mechanisms.

The simulations of the sensing performance of ring resonator chemical vapor sensors based on a four-layer Mie model have been carried out by Sun et al. [177]. Two ring resonator configurations were investigated, where a vapor sensitive polymer layer was coated on either the interior or exterior surface of a fused silica cylindrical ring resonator. Due to the interaction between the polymer and the vapor analyte, the change in RI and the polymer layer thickness lead to a spectral shift in the resonance modes. The RI sensitivity and thickness sensitivity were studied as a function of the polymer coating thickness, external RI, ring resonator size, wall thickness, resonant mode order and polarization. This study provides an insight into the WGM interaction with vapor molecules and enables sensor optimization for various applications. This proposal was also experimentally demonstrated by Sun et al. [178], with chemical-vapor sensors based on opto-fluidic ring resonators. The inner surface of the ring resonator was coated with a vapor-sensitive polymer. A MNF was used to couple the light from a 1550 $\mathrm{nm}$ tunable diode laser into the WGM. Vapor molecules passing through the ring resonator interacts with the polymer material, and subsequently changes its RI, leading to a WGM spectral shift. The ring resonator exhibits sub-second detection and recovery time with a flow rate of only 1 $\mathrm{mL} / \mathrm{min}$. The DL was estimated to be $5.6 \times 10^{-6} \mathrm{RIU}$, which can further reduced with a thicker polymer layer. Ethanol and hexane vapors were used as the model system, producing a DL for ethanol vapor of $200 \mathrm{ppm}$ in concentration or 1 $\mathrm{ng}$ in total mass.

Microspheres have also been widely used for biological detection, as shown in Fig. (10). A microsphere resonator with Q-factor of $\sim 2 \times 10^{6}$ was used as the biosensor and its sensitivity was measured using adsorption of bovine serum albumin dissolved in phosphate buffered saline on the microsphere bathed in phosphate buffered saline solution. Vollmer et al. [179] showed that microspheres can also be used to detect streptavidin binding to biotin. Noto et al. [55] characterized a nano-layer formation of $<150 \mathrm{~nm}$ on a silica microsphere in an aqueous environment by simultaneously tracking the shifts of WGMs at two wavelengths. A portion of the fiber was acid-eroded down to $3 \mu \mathrm{m}$ diameter for coupling to the WGMs of a silica microsphere. By monitoring the resonator wavelength shift at two different wavelengths $\left(\lambda_{1}=760\right.$ $\mathrm{nm}$ and $\lambda_{2}=1310 \mathrm{~nm}$ ), in-situ monitoring of bovine serum albumin monolayers with thickness in the region of $100 \mathrm{~nm}$ was achieved. With this method, not only the growth of nano-layers was monitored, but also the optical dielectric constant for the resulting film could be determined. The perturbation of an optical microsphere resonator can be used for high-sensitivity, label-free DNA quantification. The use of multiple spheres coated first with a dextran-biotin hydrogel and then with a mixture of biotinylated 27-mer oligonucleotides and streptavidin allowed for the successful detection of selected DNA strands by Vollmer et al. [180]. The hybridization to the target DNA leads to a red shift of the optical resonance wavelength and each microsphere can be identified by its unique resonance wavelength. The multiplexed signal from two microspheres allows discrimination of a single nucleotide mismatch in an 11-mer oligonucleotide with a high signal-to-noise ratio of 54. A single Influenza A virus sensor based on a microsphere resonator was later reported by Vollmer et al. [181]. An equatorial WGM of a microsphere was excited in an aqueous solution by evanescent coupling to a MNF. Resonance positions were detected as dips $\sim 5 \mathrm{pm}$ wide in the transmission spectrum and its shift enabled the detection of a single virus. The use of a microsphere with radius of $39 \mu \mathrm{m}$ and light at a wavelength $\lambda=$ $763 \mathrm{~nm}$ made it possible to detect a single virion with its mass $\sim 5.2 \times 10^{-16} \mathrm{~g}$. Detection of single cylindrical bacteria was carried out by Ren et al. [182] using surface adsorption and the induced resonance bandwidth broadening due to scattering losses. The resonance frequency was found to shift linearly with the increase of bacteria surface density. The sensitivity of the sensing system was measured to be $1.2 \times 10^{2}$ E.coli $/ \mathrm{mm}^{2}$, which corresponds to $\sim 34 \mathrm{pg} / \mathrm{mm}^{2}$ dry-mass loading or a total of 44 bacteria bound to the sphere surface at random locations.

Toroidal micro-resonators can provide extremely high Q, thus they have an obvious application in sensing. Such structures have been used for distinguishing chemically similar species. The Q-factor in heavy water $\left(\mathrm{D}_{2} \mathrm{O}\right)$ is different from that in common water $\left(\mathrm{H}_{2} \mathrm{O}\right)$ since $\mathrm{H}_{2} \mathrm{O}$ has a stronger absorption than $\mathrm{D}_{2} \mathrm{O}$. By monitoring the Q-factor, concentrations of $0.00001 \%$ of $\mathrm{D}_{2} \mathrm{O}$ in $\mathrm{H}_{2} \mathrm{O}$ have been detected by Armani et al. [56]. Another MNF coupled micro-toroid cavity resonator was reported by Zhu et al. [57] for the detection of single nanoparticles down to $30 \mathrm{~nm}$ in radius. The detection scheme is based on scattering-induced mode splitting of a WGM in an ultrahigh-Q micro-toroid. Self-referencing is made possible due to the generation of two standing wave modes upon the adsorption of a particle, improving the noise immunity of the detection system.

\subsubsection{Gas Sensors}

One of the first demonstrations of using MNFs for sensing gaseous elements was an oxygen sensor by Rosenzweig et al. [183]. The operating principle is based on the fluorescence quenching of tris-(1,10-phenanthroline)ruthenium(II) chloride in the presence of oxygen or dissolved oxygen. The $\mathrm{Ru}$ compound was implanted in an acrylamide polymer attached to a silanized 0.1-0.5 $\mu \mathrm{m}$ diameter MNF tip by photoinitiated polymerization. The minimum sample volume required for measurements was $100 \mathrm{fL}$. An absolute DL of $1 \times 10^{-17} \mathrm{M}$ was achieved, with response times less than $1 \mathrm{~s}$. Villatoto et al. [184] deposited a thin film of palladium along the uniform waist of a tapered multimode fiber. Palladium converts into palladium hydride when exposed to hydrogen, changing the RI of the external medium and thus the absorption of the evanescent field. MNFs of various diameters ranging from 30-60 $\mu \mathrm{m}$ and $10 \mathrm{~mm}$ length were coated with a $14 \mathrm{~nm}$ thick palladium film. It was found that decreasing the MNF diameter increases the signal amplitude. The average $\tau$ was $30 \mathrm{~s}$. By reducing the interaction length to $2 \mathrm{~mm}$ and the MNF diameter to $650 \mathrm{~nm}$, the response time was improved to $\tau=10 \mathrm{~s}$ [185]. Functionalized polymer MNFs for gas detection were reported by $\mathrm{Gu}$ et al. [9], which include poly-(methyl methacrylate) (PMMA), polystyrene (PS), polyacrylamide (PAM), and polyaniline/polystyrene (PANI/PS) nanowires. A $250 \mathrm{~nm}$ diameter PANI/PS MNF 
for sensing $\mathrm{NO}_{2}$ was fabricated from a polymer-blend solution. It was found from the spectral absorption that the oxidation degree of the MNF increases with the concentration of $\mathrm{NO}_{2}$. A DL below $0.1 \mathrm{ppm}$ and $\tau=7 \mathrm{~s}$ has been achieved. For sensing $\mathrm{NH}_{3}$, a $270 \mathrm{~nm}$ diameter bromothymol-blue (BTB) doped PMMA was used as the sensor head. As the $\mathrm{NH}_{3}$ gas diffuses into the MNF, the BTB reacts with $\mathrm{NH}_{3}$ and changes from acidic form to basic form, resulting in evident absorption of the probing light. A DL of better than 14 ppm has been demonstrated with $\tau=1.8 \mathrm{~s}$. By employing a dual-wavelength measurement technique, the selective detection of $\mathrm{NH}_{3}$ and humidity in gas mixtures was made possible with a PANI/PS MNF [186]. The underlying mechanism involves different levels of sensitivity to $\mathrm{NH}_{3}$ in the presence of $\mathrm{RH}$ at long and short wavelengths. MNFs coated with $\mathrm{PdAu}$ nanoparticles have shown reversible transmission changes when exposed to low concentrations of hydrogen gas (up to 8\%). Monzón-Hernández et al. [187] deposited a $0.6 \mathrm{~nm}$ thick Au layer followed by a $1.4 \mathrm{~nm}$ thick Pd layer on MNFs with diameters between 5-10 $\mu \mathrm{m}$ and lengths between 1-8 mm. Response times between 2-6 s were observed. Gas flowmetry with MNF based transducers was investigated by Lee et al. [188]. As a $188 \mu \mathrm{m}$ diameter, 9 $\mathrm{mm}$ length Poly(dimethylsiloxane) (PDMS) MNF exhibits good elastic properties, a straightened MNF was first placed between two circular apertures and then exposed to gas flow: the resulting fluidic drag force bent the MNF and displaces the output beam, which was translated into a decrease in the output power. A sensitivity of $9.8 \mathrm{~dB} / \mathrm{SLM}$ was obtained. This scheme can also be extended to measure the flow rates of liquids.

\subsection{Temperature Sensors}

Over the past few decades, temperature sensing has matured into a profound technology which has been successfully deployed in many industrial sectors, including ecological monitoring, fire detection, leakage detection, oil and gas exploration, plant and process monitoring, power cable and transmission line monitoring, storage tanks and vessels, and structural health monitoring. The compactness of MNF based structures in conjunction with its inherent immunity to electromagnetic interference has recently attracted considerable interest.

A simple modal interferometer was constructed by Zhu et al. [189], by coating the waist region of a MNF (4 mm uniform, $8 \mathrm{~mm}$ total length) with a high thermo-optic coefficient material. The ambient temperature has a great influence on the RI of the coating film and thus the interference pattern. The optical response was studied under different MNF diameters varying from $1.6 \mu \mathrm{m}$ to $20 \mu \mathrm{m}$ and a temperature OR of $-20-80{ }^{\circ} \mathrm{C}$. Veilleux et al. [190] pursued a systematic investigation on the thermal and optical properties of lyotropic liquid crystal decylammonium chloride/water/ $\mathrm{NH}_{4} \mathrm{CI}$ (DACI-LLC) from $40{ }^{\circ} \mathrm{C}$ to $80{ }^{\circ} \mathrm{C}$. A method of combining this thermo-sensitive medium and fiber tapers was proposed and studied. A different way of detecting ambient temperature changes was demonstrated by Zhang et al. [191]. A MNF was coated by a sol-gel thin film entrapping quantum dots, which provided variations in photoluminescence with temperature ranging from $30{ }^{\circ} \mathrm{C}$ to $70{ }^{\circ} \mathrm{C}$. The fabricated MNFs were subjected to thermal treatments under a nitrogen flux to protect the nanoparticles from oxidation. A sensitivity of $1 \% /{ }^{\circ} \mathrm{C}$ was observed. An inline Mach-Zehnder was conceived by Lu et al. [102], using two cascaded MNFs to excite and recombine the cladding mode with the core mode. The MNFs were of $65 \mu \mathrm{m}$ diameter and $525 \mu \mathrm{m}$ length. The highest sensitivity obtained was $0.077 \mathrm{~nm} /{ }^{\circ} \mathrm{C}$ between $20{ }^{\circ} \mathrm{C}$ and $60{ }^{\circ} \mathrm{C}$. A similar design by Chen et al. [192] using tapered sections of $35 \mu \mathrm{m}$ diameter and $200 \mu \mathrm{m}$ length achieved a sensitivity of $0.081 \mathrm{~nm} /{ }^{\circ} \mathrm{C}$ in the range $22-32{ }^{\circ} \mathrm{C}$. The device can also be used to measure liquid levels. Zhang et al. [193] used femtosecond laser to fabricate an intrinsic Fabry-Perot etalon of $100 \mu \mathrm{m}$ length on the waist of a $30 \mu \mathrm{m}$ diameter MNF. For a temperature OP of $0-100{ }^{\circ} \mathrm{C}$, the sensitivity was $12 \mathrm{pm} /{ }^{\circ} \mathrm{C}$. Temperature and strain discrimination was performed by André et al. [194] using multimode interference in a tapered coreless multimode fiber. A temperature sensitivity of $16.56 \mathrm{pm} /{ }^{\circ} \mathrm{C}$ was observed for a $87 \mu \mathrm{m}$ diameter $\mathrm{MNF}$ and $13.95 \mathrm{pm} /{ }^{\circ} \mathrm{C}$ for a $25 \mu \mathrm{m}$ diameter MNF. Sensitivity to strain as high as $-23.69 \mathrm{pm} / \mu \varepsilon$ was achieved for a 15 $\mu \mathrm{m}$ diameter MNF. The device is capable of simultaneous measurement of temperature and strain with resolutions of 1.6 ${ }^{\circ} \mathrm{C}$ and $5.6 \mu \varepsilon$ respectively. Lu et al. [101] proposed temperature sensing using a tapered bend-resistant fiber with different phase shifts between the inner and outer cladding modes. The temperature sensitivities are $-0.0253 \mathrm{rad} /{ }^{\circ} \mathrm{C}$ and $-0.0523 \mathrm{rad} /{ }^{\circ} \mathrm{C}$ respectively. Ji et al. [195] proposed a multiplexed optical temperature sensing system using MNFs with Bragg gratings as end reflectors. Experimental results showed a sensitivity of $9.7 \mathrm{pm} /{ }^{\circ} \mathrm{C}$ between $20-70{ }^{\circ} \mathrm{C}$.

Ding et al. [196] demonstrated a compact MNF coupler tip capable of sensing temperature up to $1283{ }^{\circ} \mathrm{C}$ with a 2dimensional resolution of $<200 \mu \mathrm{m}$. The device exploited the temperature dependence of intermodal coupling in the coupler uniform waist region, exhibiting a sensitivity of 11.96 $\mathrm{pm} /{ }^{\circ} \mathrm{C}$ and $\mathrm{DL}$ of $0.836^{\circ} \mathrm{C}$ when the wavelength resolution is $10 \mathrm{pm}$. Kou et al. [197] presented an all-silica high temperature sensor based on a reflective Fabry-Perot modal interferometer. A micro-cavity of $4.4 \mu \mathrm{m}$ was fabricated into a section of MNF tip with less than $10 \mu \mathrm{m}$ diameter. The interference between the fundamental mode and excited highorder modes features a temperature sensitivity of $20 \mathrm{pm} /{ }^{\circ} \mathrm{C}$ between $19-520{ }^{\circ} \mathrm{C}$, and a DL of $0.58{ }^{\circ} \mathrm{C}$ when the wavelength resolution is $10 \mathrm{pm}$. A similar temperature probe using a first-order FBG instead of a micro-cavity was presented by Kou et al. [198]. The 61-period FBG was of 6.5 $\mu \mathrm{m}$ diameter and $36.6 \mu \mathrm{m}$ length, fabricated by focused ion beam machining a MNF tip with $200 \mathrm{~nm}$ deep shallow grooves. The probe demonstrated a similar sensitivity of 20 $\mathrm{pm} /{ }^{\circ} \mathrm{C}$ operating up to $500{ }^{\circ} \mathrm{C}$. Another device combining a MNF tip and FBG was reported by Feng et al. [199]. This time, an 11-period FBG of $12 \mu \mathrm{m}$ length was engraved in a 5 $\mu \mathrm{m}$ diameter MNF tip by focused ion beam milling. The grating exhibited a temperature sensitivity of $22 \mathrm{pm} /{ }^{\circ} \mathrm{C}$.

An experimental investigation on tapered PCF coated with a layer of liquid crystal was presented by Rajan et al. [200]. Several in-line interferometers were fabricated by tapering a small section of PCF by collapsing the air holes and thinning down the collapsed region to micron-scale. Temperature tuning of the spectral response of the tapered interferometers with different waist diameters coated with high and low index liquid crystal materials were carried out. 


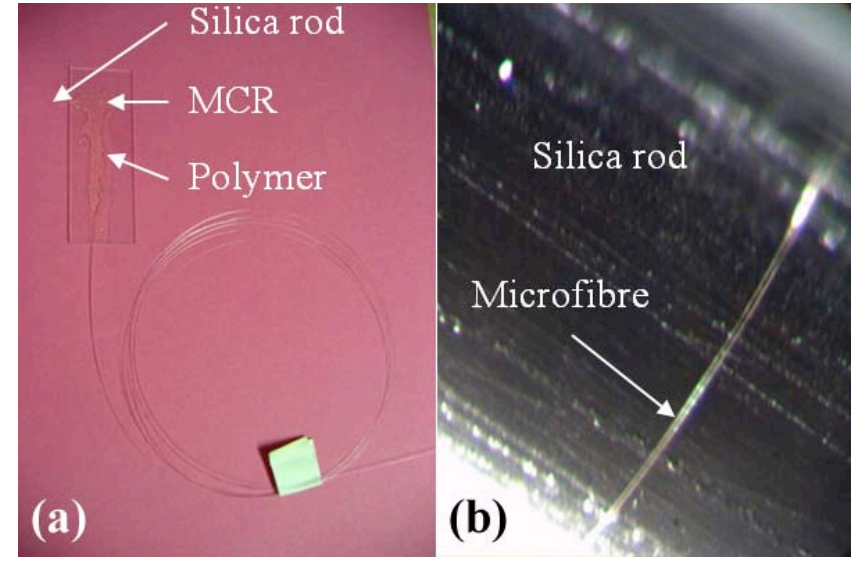

Fig. (11a). Photograph of a packaged MCR sensor, and (b) closeup view on the MNF coiled around the support rod.

Semenova et al. [201] fabricated a MNF coupler with $6 \mu \mathrm{m}$ diameter overlaid with a liquid crystal layer whose RI can be temperature-tuned. A sensitivity of $0.2 \mathrm{~nm} /{ }^{\circ} \mathrm{C}$ was shown for an OR of $50-78^{\circ} \mathrm{C}$.

In the case of MLRs, the positions of the transmission resonances are very sensitive to variations in the effective RI and circumferential length of the micro-ring, which are affected by the surrounding temperature. A temperature sensor has been reported by Sumetsky et al. [30] in a free-standing MLR, which has a larger interfacial contact area with the ambient environment than a micro-ring resonator mounted on a substrate. By monitoring the changes in the transmitted power at a wavelength near resonance, temperature variations of $0.4{ }^{\circ} \mathrm{C}$ were measured with a fast response time of $\tau=3 \mathrm{~ms}$ due to the extremely low thermal mass. The temperature DL of this temperature sensor could be as small as $\sim 0.1 \mathrm{mK}$. Harun et al. [202] presented an embedded MLR temperature sensor. The extinction ratio of the transmission spectrum was linearly proportional to the ambient temperature and the slope of the extinction ratio reduction against temperature was calculated to be $0.043 \mathrm{~dB} /{ }^{\circ} \mathrm{C}$.

MKRs have been reported for temperature sensing by $\mathrm{Wu}$ et al. [203]. An $\mathrm{MgF}_{2}$ crystal plate was adopted as substrate and sensing knots were covered by a thin $\mathrm{MgF}_{2}$ slab to keep it steady and immune to environmental fluctuations. A thermally induced resonance wavelength shift resulting in a sensitivity of $52 \mathrm{pm} /{ }^{\circ} \mathrm{C}$ was recorded in the range $30-700{ }^{\circ} \mathrm{C}$ with a temporal response of $\tau \sim 1 \mathrm{~ms}$ for a silica sensor MKR. A polymer based MKR sensor showed a sensitivity of $266 \mathrm{pm} /{ }^{\circ} \mathrm{C}$ within $20-80{ }^{\circ} \mathrm{C}$ and a response time of $\tau=5 \mathrm{~ms}$. Better performance can be realized by improving the Qfactor of the MKR. More recently, the same research group presented theoretically and experimentally a high precision and simultaneous multi-point temperature sensor, by cascading two MKRs and measuring the shift difference between the first and second-order resonance peaks [204]. Zeng et al. [205] studied an embedded silica MKR coated with low refractive index fluoro-polymers (Efiron UV-373) that provided sensitivities as high as $0.28 \mathrm{~nm} /{ }^{\circ} \mathrm{C}$ in the temperature OR of $25-135{ }^{\circ} \mathrm{C}$, which corresponds to a DL of $0.5^{\circ} \mathrm{C}$.

MCRs embedded in the Teflon (Fig. 11) with opposite thermo-optic coefficients were proposed and demonstrated as a temperature-insensitive device by Chen et al. [206]. By

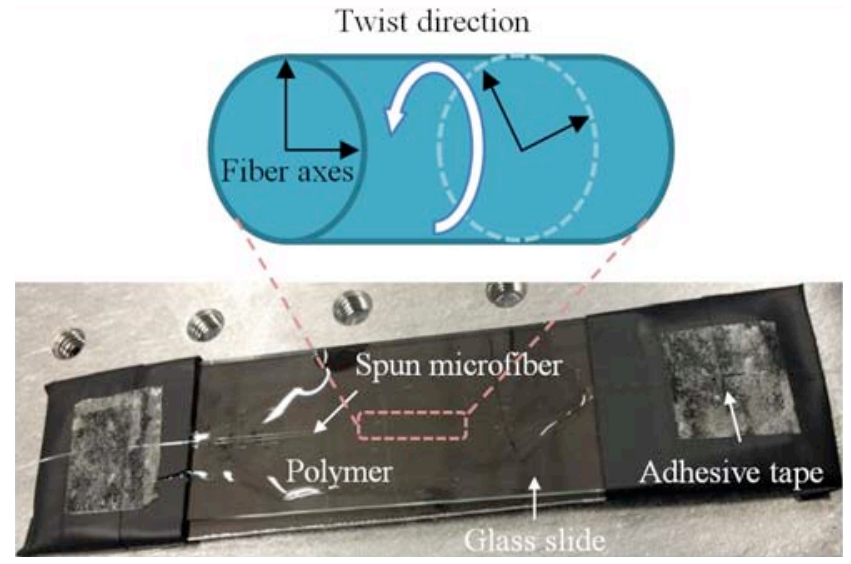

Fig. (12). Photograph and schematic diagram of spun MNF embedded in polymer for characterization.

coiling three turns of MNF of $3 \mu \mathrm{m}$ diameter, a sensitivity of $<6 \mathrm{pm} /{ }^{\circ} \mathrm{C}$ was achieved in the room temperature range. It was found that sensitivity can be optimized by tuning the MNF diameter. Chen et al. [207] also showed the temperature sensing capability of Teflon-embedded MCRs with a demonstration using MNF of 4-5 $\mu \mathrm{m}$ diameter and $14 \mu \mathrm{m}$ length, coiled around a PMMA rod of $2 \mu \mathrm{m}$ diameter. The sensitivity to temperature was observed to be $80 \mathrm{pm} /{ }^{\circ} \mathrm{C}$. The application of MCRs as a tool for the rapid inspection of electrical wires for insulation faults and current surges was demonstrated by Chen et al. [208]. The MCR was integrated on a sliding probe that maps the local temperature to identify positions with insulation faults that can result in electrical arcing. Defects can be distinguished by an increase in heat signature. The ring-shaped detection area facilitates rotationally symmetric coverage and thus removes the need for radial alignment of the sensor head. A sensitivity of $95 \mathrm{pm} /{ }^{\circ} \mathrm{C}$ from $26{ }^{\circ} \mathrm{C}$ to $76{ }^{\circ} \mathrm{C}$ was demonstrated with a MNF of $2 \mu \mathrm{m}$ diameter and $15 \mathrm{~mm}$ length, coiled around a polymer-coated Teflon capillary of $1.8 \mathrm{~mm}$ diameter.

\subsection{Current Sensors}

There are well known advantages in using optical current sensors over conventional current transformers. Due to the dielectric nature of optical fibers, its sensors have the robustness to take measurements in high voltage or high magnetic induction noise fields and immunity from saturation effects that otherwise may limit conventional current transformers. In addition, optical current sensors have a high linear response over a wide frequency bandwidth that allows them to detect transient electrical faults, inspect noise on direct current (DC) lines for the monitoring of partial discharges during automatic control, and protection of high power equipment and vital electrical components. Their compactness, lightweight and potentially available at low-cost makes them an attractive choice as sensors.

The short optical path length of coiled MNFs leads to a high operating bandwidth compared to regular fiber coils, which are much bulkier due to the minimum bend radius restriction. Belal et al. introduced coiled MNFs as a compact current sensors, by wrapping a MNF around a conductive wire. Alternating currents can be detected by either a polarimetric technique via the Faraday Effect [27], or by track- 
ing thermally induced phase shifts when incorporated into the sensing arm of an interferometer [209]. For the former configuration, a sensitivity of $16.8 \mu \mathrm{rad} / \mathrm{A}$ was achieved for a 25 -turn device. For the latter configuration, the measured sensitivity was $1.28 \times 10^{-4} \mathrm{rad} / \mathrm{I}^{2}$. In both cases, the MNF was of $5 \mu \mathrm{m}$ diameter, $\sim 10 \mathrm{~cm}$ length, and the sensitivity was expected to increase with the number of MNF turns. However, birefringence played a major factor in the quality of fabricated micro-coil sensor heads. The net power transfer between the fast and slow axes due to Faraday rotation is maximum after quarter of a beat length, and reduces to zero over half of a beat length. In order to improve the general sensitivity and reproducibility of the samples, spun MNF was introduced by Chen et al. [210], as shown in Fig. (12). It was found that spinning a side-polished optical fiber during the tapering process packs enough intrinsic linear birefringence to resist bend- and packaging-induced birefringence, and sufficient circular birefringence to support efficient Faraday rotation.

By wrapping MLR around a copper rod, the resonance wavelength can be tuned by applying an electric current through the copper rod. For a MLR with Q-factor of 4000, an approximately linear dependence of wavelength shift on the electric current was demonstrated by Guo et al. [37] with a slope of $26.5 \mathrm{pm} / \mathrm{A}$. Using similar principles, Lim et al. [211] achieved current sensing by wrapping a MKR around a copper wire. The wavelength shift was measured to be linearly proportional to the square of the current and a maximum tuning slope of $51.3 \mathrm{pm} / \mathrm{A}^{2}$ was obtained. MLRs were theoretically investigated by Chen et al. [212], and were found to exhibit optimum geometries that could host efficient Faraday rotation despite the presence of birefringence. A set of design rules were established for manufacturing non-ideal MLR sensor heads with a high sensitivity to current.

MCRs have also been exploited for current sensing applications by Chen et al. [213]. Due to the increased optical path length under resonant conditions with respect to the actual physical dimension, an MCR with a Q-factor of 17000 exhibited a factor of 3.1 enhancement in Faraday rotation between on- and off- resonance. Sharper resonances and lower loss were expected to considerably improve the sensitivity of the MCR. To improve the optical stability of MCR based sensors from environmental effects, Chen et al. [214] proposed two stabilization techniques. The passive technique involves a redesign of the typical geometry of MCRs by chirping the RI of successive pairs of turns to widen the resonance shape. The active technique utilizes a piezoceramic transducer as the support disc of the MCR, which can tune its geometry to compensate for drifts in the resonant wavelength. Sulaiman et al. [215] demonstrated a MachZehnder structure with MNF coiled around a copper wire in the sensing arm. As direct current (DC) current passes through the MNF-wound inductor, thermal effects induce a spectral shift of the interference pattern. Experimental results indicated maximum tuning slope of $0.54 \mathrm{~nm} / \mathrm{A}^{2}$, between $I=$ $0 \mathrm{~A}$ and $2.5 \mathrm{~A}(\mathrm{DC})$.

\subsection{Displacement Sensors}

This category of sensors can be found in a wide variety of industrial applications including semiconductor process- ing, assembly of disk drives, precision thickness measurements, machine tool metrology and assembly line testing. The aforementioned characteristics of MNF based structures enable such optical sensors to operate in confined spaces.

The bending effects in MNFs were experimentally investigated by Arregui et al. [216] to yield a set of design rules for implementing displacement sensors. In their work, the MNF diameters ranged from $22 \mu \mathrm{m}$ to $66 \mu \mathrm{m}$. It was found that narrower waist diameters lead to a higher spatial resolution, whilst wider waist diameters enabled a broader measurement range. A locally bent MNF bimodal interferometer was presented by Luo et al. [217] as a displacement sensor, based on spectral shifts resulting from the beating between the fundamental mode and the first excited high-order mode. The MNF diameter was carefully chosen at $1.92 \mu \mathrm{m}$ such that the thermo-optic coefficients of the two modes are equal, leading to temperature-insensitive measurements. The optical sensitivity to micro-displacements was $102 \mathrm{pm} / \mu \mathrm{m}$. Ji et al. [195] demonstrated the feasibility of a multiplexed optical displacement sensing system using cascaded MNFBragg-gratings. The fiber taper of each sensing branch acted as sensor head to provide a displacement-induced curvaturedependent bend loss. The FBG provided the end-reflection that identified the particular sensor head with a unique Bragg wavelength. The resulting optical signals were then distinguished by their wavelength and the displacement in each case was proportional to the individual peak intensity. Experimental results showed a sensitivity of $0.11 \mathrm{~dB} / \mu \mathrm{m}$ for displacements within an OR of 0-400 $\mu \mathrm{m}$. Yoon et al. [218] proposed and fabricated a grating structure using a periodic arrangement of $76 \mu \mathrm{m}$ diameter MNFs with $1.3 \mathrm{~mm}$ separation. By analysing the resonant wavelength shift and intensity variations resulting from mode coupling effects, it is possible to distinguish between the sensitivities to displacement-induced strain $(-0.55 \mathrm{~nm} / \mathrm{m} \varepsilon,-0.32 \mathrm{~dB} / \mathrm{m} \varepsilon)$ and temperature $\left(49.6 \mathrm{pm} /{ }^{\circ} \mathrm{C},-0.01 \mathrm{~dB} /{ }^{\circ} \mathrm{C}\right)$.

As previously mentioned, the MLR transmission spectrum is strongly dependent on the loop size. As a result, they have been exploited as displacement sensors by MartinezRios et al. [219]. Typically, one of the loop pigtails was attached to the moving surface. The principle of operation is based on the interaction between the fundamental cladding mode propagating through the MNF waist and the excited higher order cladding modes when the MNF is deformed to form a loop. The notch wavelength resonances shift as a function of the loop diameter. A sensitivity of $0.116 \mathrm{~nm} / \mu \mathrm{m}$ has been achieved in a displacement OR of $3.125 \mathrm{~mm}$ and the maximum wavelength shift was $360.93 \mathrm{~nm}$. Alternatively, a sensitivity of $2.7 \mathrm{nW} / \mu \mathrm{m}$ was recorded when the measurements were carried out around a center wavelength of $\lambda=1280 \mathrm{~nm}$ with a low power LED source of $100 \mathrm{~nm}$ bandwidth and a Ge-based photodetector. A micro-ball lens structure fabricated at the cleaved tip of a MNF coupler was demonstrated for displacement measurements by Jasim et al. [220]. The diameter of the MNF and micro-ball measures 40 $\mu \mathrm{m}$ and $102 \mu \mathrm{m}$ respectively. The device operates on the interference between the reflected beams from the micro-ball lens and the reflector, with an increased Q-factor for larger displacements. 


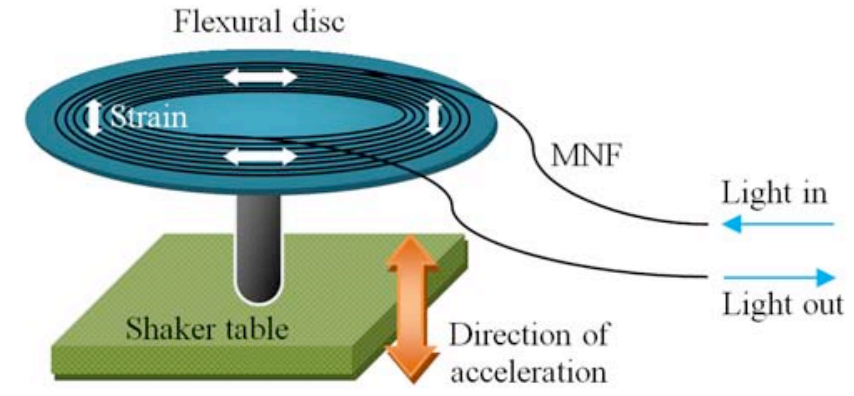

Fig. (13). Illustration of the MNF based flexural disc accelerometer operating under acceleration.

\subsection{Bend Sensors}

Bend or curvature sensors have various applications in the areas of mechanical engineering, robotic arms, structural health monitoring and turbulence sensing. With immunity to electromagnetic interference and compact size, the advantages of MNF based devices couldn't be clearer.

A compact curvature sensor based on concatenating two MNFs of $60 \mu \mathrm{m}$ diameter has been demonstrated by Monzon-Hernandez et al. [221]. When the device is bent, the symmetry of the straight taper is lost and light from the first taper excites cladding modes. In the second taper, a fraction of the light guided by the cladding modes couples back to the fundamental mode, producing an interference pattern in the transmission spectrum. Wang et al. [222] established a technique for creating periodic tapers in an lead silicate softglass fiber using a focused $\mathrm{CO}_{2}$ laser beam. The bend sensing ability of the periodically tapered structure of $140 \mu \mathrm{m}$ diameter and $390 \mu \mathrm{m}$ pitch was also investigated. A sensitivity of $-27.75 \mu \mathrm{W} / \mathrm{m}^{-1}$ was achieved by means of measuring the bend-induced change in light intensity. A vector bend sensor was proposed by Zhang et al. [223], consisting of a lateral-offset splicing joint and an up-taper formed through an excessive fusion splicing method. The diameter and length of the expanded section are $168 \mu \mathrm{m}$ and $280 \mu \mathrm{m}$ respectively, with a lateral-offset of $6.5 \mu \mathrm{m}$. At the first lateraloffset fusion-splicing joint, the input light is split into fundamental core and cladding modes. The cladding modes couples back into the core when propagating through the uptaper, forming a MZI due to the phase difference between the core and cladding modes. For a curvature OR from $-3 \mathrm{~m}^{-1}$ to $3 \mathrm{~m}^{-1}$, the bend sensitivities at $1463.86 \mathrm{~nm}$ and $1548.41 \mathrm{~nm}$ are $11.987 \mathrm{~nm} / \mathrm{m}^{-1}$ and $8.697 \mathrm{~nm} / \mathrm{m}^{-1}$ respectively. Wu et al. [224] reported the construction of an in-line MZI by combining a MNF with a LPG in a section of all-solid photonic bandgap fiber. The MNF was of $94.5 \mu \mathrm{m}$ and $350 \mu \mathrm{m}$ length. The LPG had a pitch of $160 \mu \mathrm{m}$ and a length of $6.40 \mathrm{~mm}$. The sensing mechanism can be attributed to mode coupling caused by bandgap-shifting as the fiber diameter is abruptly scaled down.

\subsection{Surface Sensors}

The detection and minimization of surface and bulk nonuniformities of MNFs are of critical importance for reducing their optical loss during fabrication. These sensing techniques can be extended for quality control of regular and special types of optical fibers.
Birks et al. [225] devised and tested a simple way of measuring the diameter uniformity of optical fibers. The proposed method uses a MNF as the probe, which translates across small distances along the fiber under test and periodically touches it for discrete measurements. At these points, the MNF transmission spectrum exhibits whispering-gallerymode resonances that shift in wavelength depending on the effective radius of the local region of fiber. Scanning MNF surface and bulk distortions with sub-nanometer accuracy was developed by Sumetsky et al. [226]. Their method employs a partially stripped single-mode fiber as a probe that slides along a MNF transmitting the fundamental mode. The intensity of light absorbed by the probe depends on the local distribution of the mode propagating in the MNF. By measuring variations in the transmittance of the probe output, the uniformity of the MNF can be determined. Sumetsky et al. [227] also improved upon the first demonstration by Birks et al., to reach a DL of angstrom-scale. A phase-locked loop based topography measurement of ultra-precision machined surface was presented by Lee et al. [228] using ball-lensed and tapered-lensed fibers. Although interferometric fringes were not found in the case of the ball-lensed fiber, the machined surface could be measured by autofocusing and intensity methods. On the other hand, it was found that phaselocked loop based Fizeau interferometry can be applied to measure the surface profile with the tapered-lensed fiber.

\subsection{Acceleration Sensors}

Accelerometers are well established in the commercial landscape. They are employed in earthquake monitoring, guidance systems, inertial navigation, platform stabilization, vibration monitoring in portable electronics, machinery, vehicles and vessels. Such optical sensors are highly desirable since they are unaffected by electromagnetic interference from static electricity, strong magnetic fields and surface potentials.

A MNF based flexural disc was first suggested and demonstrated by Chen et al. [229]. Conventional flexural disc accelerometers are well known for their relatively high bandwidth and high sensitivity that is proportional to the disc radius. Axial acceleration induces extensive and compressive strain in the MNF depending on the phase of motion, as shown in Fig. (13). This translates to a phase modulation that can be measured interferometrically. However, for applications requiring compact solutions, such sensors cannot perform adequately. The minimum bend radius of MNFs allows very small device dimensions to be feasible without the problems of bend loss or depolarization. In addition, the low volume-tolength ratio of MNFs reduces the load during the flexing motion of the disc and thus enables higher sensitivity. The influence of design parameters was studied and an efficient surface winding technique for the MNF was proposed [230]. A sensitivity of $4 \mathrm{rad} / \mathrm{g}$ has been demonstrated for a $60 \mathrm{~mm}$ length MNF embedded on a $25 \mathrm{~mm}$ diameter flexural disc composed of pyrolytic graphite. The performance of the sensor can be improved with longer lengths of MNF.

A MNF based vibration sensor based on an optical trapping mechanism was proposed by Wang et al. [231]. A polystyrene microsphere with a diameter of $10 \mu \mathrm{m}$ was used as 
the mass block, enclosed in a sensing cavity filled with refractive index matching liquid. Two MNFs were inserted such that vibration-induced location variations of the microsphere will change the amount of light collected by the MNF tips. An OR of $15-1000 \mathrm{~Hz}$ was demonstrated in the preliminary results.

High Q-factor MLRs and MKRs have also been demonstrated as accelerometers by Hou et al. and Wu et al. [232, 233 ] in the OR of $20 \mathrm{~g}$. The resonators were individually fixed on to the surface of a cantilever beam and attached to a micro-machined silicon proof mass. The MLR based sensor ( $1 \mu \mathrm{m}$ diameter, $250 \mu \mathrm{m}$ loop diameter) provided a sensitivity of $624.7 \mathrm{mV} / \mathrm{g}$, while the MKR based sensor $(1.1 \mu \mathrm{m}$ diameter, $386 \mu \mathrm{m}$ knot diameter) reached a slightly higher performance of $654.7 \mathrm{mV} / \mathrm{g}$.

\subsection{Force Sensors}

Force sensors have gathered increasing interest over the years and have established its role in many fields such as biomechanics, civil engineering, fluid flow measurements, motor sport, process monitoring and control. For optical fiber based sensors, the sensitivity tends to scale inversely with the fiber cross-sectional area. Therefore, it is possible to increase force sensitivity by using MNFs instead of conventionally sized fibers. The small dimensions of MNFs also minimize the intrusiveness of taking sensitive measurements.

A FBG inscribed along the uniform waist of a MNF has been demonstrated by Wieduwilt et al. [234]. The forceinduced axial strain produced a shift in the Bragg wavelength, inducing a sensitivity of $1900 \mathrm{~nm} / \mathrm{N}$ for a $3.5 \mu \mathrm{m}$ diameter, $8 \mathrm{~mm}$ length MNF. Forces as small as $25 \mu \mathrm{N}$ could be measured with this sensor. The thermal and mechanical properties of MNFs were investigated by Wang et al. [235]. It was found that the temperature or strain induced spectral shifts are strongly related to the RI change of the fundamental mode. A MNF of $6 \mu \mathrm{m}$ diameter and $25 \mathrm{~mm}$ length resulted in a sensitivity of $620.83 \mathrm{~nm} / \mathrm{N}$. Wang et al. [236] proposed combining MNFs with a frequency-domain reflectometry technique to create a high sensitivity and high resolution force sensor. The cross-correlation wavelength shift in the uniform waist region of the MNF is related to the RI change of the fundamental mode. The force sensitivity was significantly improved due to its reduced diameter. A force sensitivity of $620.83 \mathrm{~nm} / \mathrm{N}$ and DL of $6.35 \mu \mathrm{N}$ was demonstrated with a spatial resolution of $3.85 \mathrm{~mm}$ for a MNF of 6 $\mu \mathrm{m}$ diameter, which is about 500 times higher than that of its SMF equivalent.

\subsection{Rotation Sensors}

Rotation sensors have numerous applications, notably employed in inertial navigation systems, control, stabilization, and positioning systems. Although both mechanical and optical techniques exist for rotation sensing, the latter was found to provide higher sensitivity and lower drift rates. Micro-resonators are advantageous in the sense that they can permit enhancements in the sensitivity without enlarging the dimensions of the device.

Rotation sensors and gyroscopes with enhanced sensitivities have been predicted for MCR based structures using the slow light effect by Scheuer et al. [51]. The combination of slow-light and conventional propagation effects leads to the enhancement of the rotation-detection sensitivity. Simulations estimated that for a lossless MCR, the sensitivity to rotation could improve by four orders of magnitude with respect to the case of un-optimised conventional optical fiber gyroscopes with similar parameters. However, a later theoretical analysis by Digonnet et al. [237] revealed that gyroscopes made from MCRs are actually less sensitive than conventional resonant fiber-optic gyroscopes consisting of a single loop. The sensitivity is proportional to the total group delay of light through the structure and the maximum achievable group delay is limited in the same manner by loss.

\subsection{Acoustic Sensors}

The commercial use of acoustic sensing spans several decades, and is one of the most successful applications in the field of optical fiber sensors. Rapid advances in this area have been driven by the increasing demand from industries such as defense. The acoustic signatures of tanks, aircrafts, helicopters and submarines can be monitored for high precision battlefield awareness and surveillance. MNF based devices offer less intrusion and easier deployment, making them an attractive choice.

A simple vibration sensor based on a non-adiabatic MNF was shown by $\mathrm{Xu}$ et al. [238]. The environmental vibrations were detected by demodulating the transmission loss of the $22 \mu \mathrm{m}$ diameter MNF. The sensing system displayed a wide frequency response, ranging from a few hertz to tens of kilohertz.

Chen et al. [239] proposed and demonstrated a compact microphone comprising of a $2 \mu \mathrm{m}$ diameter, $35 \mathrm{~mm}$ length MNF coiled around a $3 \mathrm{~mm}$ diameter air-backed mandrel. Acoustic waves induce local pressure variations that change the mandrel diameter and thus the optical path length of the mode propagating in the MNF. The phase modulation was detected via a single-fiber polarimetric interferometer employing balanced detection. The device in shown in Fig. (14) exhibited an average acoustic sensitivity of -137 re. $\mathrm{rad} / \mathrm{m} . \mu \mathrm{Pa}$ and $-142 \mathrm{~dB}$ re. $\mathrm{rad} / \mathrm{m} . \mu \mathrm{Pa}$ between $40-500 \mathrm{~Hz}$ and $1.5-4 \mathrm{kHz}$ respectively. The average DL measured with no noise averaging between $40-500 \mathrm{~Hz}$ and $1.5-4 \mathrm{kHz}$ are $38.9 \mathrm{~dB}_{\mathrm{SPL}}$ and $44.3 \mathrm{~dB}_{\mathrm{SPL}}$ respectively.

\subsection{Electric Field Sensors}

Optical electric field sensors have attracted the attention of several industrial segments due to the fact that they can act as dielectric receiver antennas. MNF based sensor probes have excellent performance as field receivers compared to metal-based electric field probes due to their immunity to electromagnetic interference. Such low-invasive and noiserejecting quality exhibits the ideal way of detecting of localized electric field distributions. Moreover, such sensors offer reduced power consumption and smaller size than their conventional electronic counterpart.

Veilleux et al. [240] have demonstrated control over the light transmission of a MNF using a layer of electric-fieldtunable liquid crystal (Merck, ZLI-1800-100). The MNF was 


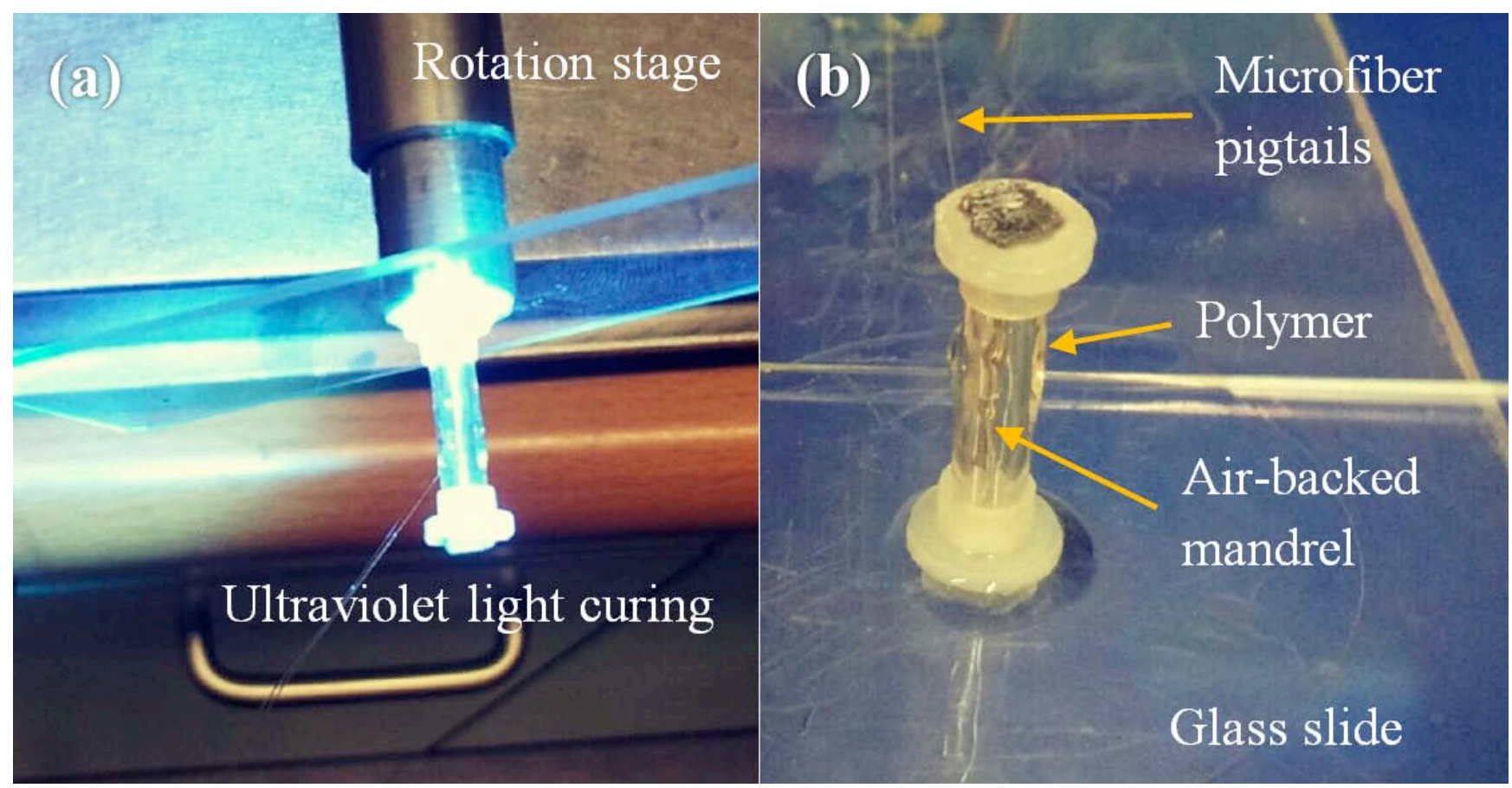

Fig. (14a). Ultraviolet-curing of the polymer coating provided robust packaging for the microphone. (b) Fabricated sample fixed on a glass slide for acoustic interrogation.

of $15 \mu \mathrm{m}$ diameter and $5 \mathrm{~mm}$ length. When the voltage applied between two electrodes changed from $0 \mathrm{~V}$ to $350 \mathrm{~V}$, the external RI changed from 1.33 to 1.48 . Such a device could be adapted into a compact electric field optical sensor.

\subsection{Magnetic Field Sensors}

Magnetic field sensors have coexisted alongside electric field sensors due to their duality transformations. Fidelity, compactness and configurability are the most important parameters for a good magnetic field sensor design. Thus, magnetic field sensing with MNF devices has inherent advantages when it comes to meeting these requirements. The broad range of possible applications includes automotive, navigation, proximity sensors, spatial and geophysical research, transducer for micro-actuators, traffic counting and vehicle detection.

A MKR was demonstrated as a magnetic field sensor by Li et al. [241]. The device was submersed in a magnetic fluid that can be modulated to influence the effective index and thus the resonant wavelength of the MKR. A linear sensitivity of $0.3 \mathrm{pm} / \mathrm{Oe}$ up to $300 \mathrm{Oe}$, and a DL of $\sim 10$ Oe was reported.

\section{CONCLUSIONS}

In conclusion, optical microfiber and nanofiber based sensors is a rapidly growing field. MNFs exhibit many desirable characteristics such as excellent optical confinement, strong evanescent field, bend-insensitivity and could be made into resonators. The resulting sensors hold numerous advantages over regular optical fiber based counterparts, including small-size, non-intrusive, selective, robustness, fast-response, high sensitivity, low detection limit and the feasibility of extremely high-Q resonators. With sensing ar- eas spanning refractive index, bio-chemical, temperature, current, displacement, bend, surface, acceleration, force, rotation, acoustic, electric field and magnetic field, the future of MNFs looks exceptionally bright and promising. We have no doubt that new applications will continue to arise from the development of MNF based sensors.

\section{CONFLICT OF INTEREST}

The authors confirm that this article content has no conflicts of interest.

\section{ACKNOWLEDGEMENTS}

Declared none.

\section{REFERENCES}

[1] Brambilla G. Optical fibre nanowires and microwires: a review. J Optics-Uk 2010; 12: 043001.

[2] Stiebeiner A, Garcia-Fernandez R, Rauschenbeutel A. Design and optimization of broadband tapered optical fibers with a nanofiber waist. Opt Express 2010; 18: 22677-85.

[3] Birks TA, Li YW. The Shape of Fiber Tapers. J Lightw Technol 1992; 10: 432-8.

[4] Tong LM, Hu LL, Zhang JJ, et al. Photonic nanowires directly drawn from bulk glasses. Opt Express 2006; 14: 82-7.

[5] Brambilla G, Koizumi E, Feng X, et al. Compound-glass optical nanowires. Electron Lett 2005; 41: 400-2.

[6] Magi EC, Fu LB, Nguyen HC, et al. Enhanced Kerr nonlinearity in sub-wavelength diameter As2Se3 chalcogenide fiber tapers. Opt Express 2007; 15: 10324-9.

[7] Harfenist SA, Cambron SD, Nelson EW, et al. Direct drawing of suspended filamentary micro- and nanostructures from liquid polymers. Nano Lett 2004; 4: 1931-7.

[8] Nain AS, Wong JC, Amon C, et al. Drawing suspended polymer micro-/nanofibers using glass micropipettes. Appl Phys Lett 2006; 89: $183105-7$.

[9] Gu FX, Zhang L, Yin XF, et al. Polymer single-nanowire optical sensors. Nano Lett 2008; 8: 2757-61. 
[10] Xing XB, Wang YQ, Li BJ. Nanofiber drawing and nanodevice assembly in poly(trimethylene terephthalate). Opt Express 2008; 16: 10815-22.

[11] Guo ML, Shi JC, Li BJ. Polymer-based micro/nanowire structures for three-dimensional photonic integrations. Opt Lett 2008; 33: 2104-6.

[12] Okamoto K. Fundamentals of optical waveguides. 2nd ed. ed. Burlington, Mass. ; London: Academic Press 2006.

[13] Tong LM, Lou JY, Mazur E. Single-mode guiding properties of subwavelength-diameter silica and silicon wire waveguides. Opt Express 2004; 12: 1025-35

[14] Wu M, Huang WQ, Wang LL. Propagation characteristics of the silica and silicon subwavelength-diameter hollow wire waveguides. Chin Opt Lett 2008; 6: 732-5.

[15] Tong LM, Gattass RR, Ashcom JB, et al. Subwavelength-diameter silica wires for low-loss optical wave guiding. Nature 2003; 426: 816-9.

[16] Tong LM, Lou JY, Gattass RR, et al. Assembly of silica nanowires on silica aerogels for microphotonic devices. Nano Lett 2005; 5 : 259-62.

[17] Lacroix S, Black RJ, Veilleux C, et al. Tapered Single-Mode Fibers - External Refractive-Index Dependence. Appl Optics 1986; 25: 2468-9.

[18] Diez A, Andres MV, Cruz JL. In-line fiber-optic sensors based on the excitation of surface plasma modes in metal-coated tapered fibers. Sens Actutors B-Chem 2001; 73: 95-9.

[19] Liang W, Huang YY, Xu Y, et al. Highly sensitive fiber Bragg grating refractive index sensors. Appl Phys Lett 2005; 86: 151122.

[20] Allsop T, Floreani F, Jedrzejewski KP, et al. Spectral characteristics of tapered LPG device as a sensing element for refractive index and temperature. J Lightw Technol 2006; 24: 870-8.

[21] Wo JH, Wang GH, Cui Y, et al. Refractive index sensor using microfiber-based Mach-Zehnder interferometer. Opt Lett 2012; 37: $67-9$.

[22] Tian ZB, Yam SSH, Barnes J, et al. Refractive index sensing with Mach-Zehnder interferometer based on concatenating two singlemode fiber tapers. IEEE Photonics Technol Lett 2008; 20: 626-8

[23] Tian ZB, Yam SSH, Loock HP. Refractive index sensor based on an abrupt taper Michelson interferometer in a single-mode fiber. Opt Lett 2008; 33: 1105-7.

[24] Ding JF, Zhang AP, Shao LY, et al. Fiber-taper seeded long-period grating pair as a highly sensitive refractive-index sensor. IEEE Photonics Technol Lett 2005; 17: 1247-9.

[25] Li J, Sun LP, Gao SA, et al. Ultrasensitive refractive-index sensors based on rectangular silica microfibers. Opt Lett 2011; 36: 3593-5.

[26] Tan WH, Shi ZY, Smith S, et al. Submicrometer Intracellular Chemical Optical Fiber Sensors. Science 1992; 258: 778-81.

[27] Belal M, Song Z, Jung Y, et al. Optical fiber microwire current sensor. Opt Lett 2010; 35: 3045-7.

[28] Bueno FJ, Esteban O, Diaz-Herrera N, et al. Sensing properties of asymmetric double-layer-covered tapered fibers. Appl Opt 2004; 43: $1615-20$.

[29] Sumetsky M, Dulashko Y, Fini JM, et al. Optical microfiber loop resonator. Appl Phys Lett 2005; 86: 161108.

[30] Sumetsky M, Dulashko Y, Fini JM, et al. The microfiber loop resonator: Theory, experiment, and application. J Lightw Technol 2006; 24: 242-50.

[31] Vienne G, Li Y, Tong L. Effect of host polymer on microfiber resonator. IEEE Photonics Technol Lett 2007; 19: 1386-8.

[32] Pal P, Knox WH. Fabrication and Characterization of Fused Microfiber Resonators. IEEE Photonics Technol Lett 2009; 21: 766-8.

[33] Wang P, Zhang L, Yang ZY, et al. Fusion Spliced Microfiber Closed-Loop Resonators. IEEE Photonics Technol Lett 2010; 22: 1075-7.

[34] Pal P, Knox WH. Low loss fusion splicing of micron scale silica fibers. Opt Express 2008; 16: 11568-73.

[35] Li W, Wang P, Hu ZF, et al. Fusion Splicing Soft Glass Microfibers for Photonic Devices. IEEE Photonics Technol Lett 2011; 23: 831-3.

[36] Guo X, Tong LM. Supported microfiber loops for optical sensing. Opt Express 2008; 16: 14429-34.

[37] Guo X, Li YH, Jiang XS, et al. Demonstration of critical coupling in microfiber loops wrapped around a copper rod. Appl Phys Lett 2007; 91: 073512 .

[38] Jiang XS, Tong LM, Vienne G, et al. Demonstration of optical microfiber knot resonators. Appl Phys Lett 2006; 88: 223501.
[39] Wang YQ, Zhu H, Li BJ. Optical characterization of mechanically tunable microwire based resonators by changing ring radius and wire diameter. Opt Commun 2011; 284: 3276-9.

[40] Sumetsky M. Optical fiber microcoil resonator. Opt Express 2004; 12: 2303-16.

[41] Vahala KJ. Optical microcavities. Nature 2003; 424: 839-46.

[42] Gorodetsky ML, Ilchenko VS. High-Q Optical Whispering-Gallery Microresonators - Precession Approach for Spherical Mode Analysis and Emission Patterns with Prism Couplers. Opt Commun 1994; 113: $133-43$

[43] Sumetsky M, Dulashko Y, Windeler RS. Super free spectral range tunable optical microbubble resonator. Opt Lett 2010; 35: 1866-8.

[44] Xu F, Brambilla G. Manufacture of 3-D microfiber coil resonators. IEEE Photonics Technol Lett 2007; 19: 1481-3.

[45] Sumetsky M. Basic elements for microfiber photonics: Micro/nanofibers and microfiber coil resonators. J Lightw Technol 2008; 26: 21-7

[46] Xu F, Brambilla G. Demonstration of a refractometric sensor based on optical microfiber coil resonator. Appl Phys Lett 2008; 92: 101126.

[47] Xu F, Horak P, Brambilla G. Conical and biconical ultra-high-Q optical-fiber nanowire microcoil resonator. Appl Optics 2007; 46: 570-3.

[48] Xu F, Wang Q, Zhou JF, et al. Dispersion Study of Optical Nanowire Microcoil Resonators. IEEE J Select Top Quant Electron 2011; 17: 1102-6.

[49] Xu F, Brambilla G, Feng J, et al. Mathematical model for manufacturing microfiber coil resonators. Opt Eng 2010; 49: 044001.

[50] Scheuer J, Sumetsky M. Optical-fiber microcoil waveguides and resonators and their applications for interferometry and sensing. Laser Photon Rev 2011; 5: 465-78.

[51] Scheuer J. Fiber microcoil optical gyroscope. Opt Lett 2009; 34: 1630-2.

[52] Hsieh YC, Peng TS, Wang LA. Millimeter-Sized Microfiber Coil Resonators With Enhanced Quality Factors by Increasing Coil Numbers. IEEE Photon Technol Lett 2012; 24: 569-71.

[53] Jasim AA, Lim KS, Muhammad MZ, et al. Transmission characteristic of multi-turn microfiber coil resonator. Opt Laser Technol 2012; 44: 1791-5.

[54] Brambilla G, Murugan GS, Wilkinson JS, et al. Optical manipulation of microspheres along a subwavelength optical wire. Opt Lett 2007; 32: 3041-3.

[55] Noto M, Vollmer F, Keng D, et al. Nanolayer characterization through wavelength multiplexing of a microsphere resonator. Opt Lett 2005; 30: 510-2.

[56] Armani AM, Vahala KJ. Heavy water detection using ultra-high-Q microcavities. Opt Lett 2006; 31: 1896-8.

[57] Zhu JG, Ozdemir SK, Xiao YF, et al. On-chip single nanoparticle detection and sizing by mode splitting in an ultrahigh-Q microresonator. Nat Photon 2010; 4: 46-9.

[58] White IM, Gohring J, Sun Y, et al. Versatile waveguide-coupled optofluidic devices based on liquid core optical ring resonators. Appl Phys Lett 2007; 91: 241104.

[59] Sumetsky M, Windeler RS, Dulashko Y, et al. Optical liquid ring resonator sensor. Opt Express 2007; 15: 14376-81.

[60] Murugan GS, Wilkinson JS, Zervas MN. Selective excitation of whispering gallery modes in a novel bottle microresonator. Opt Express 2009; 17: 11916-25

[61] Ding M, Murugan GS, Brambilla G, et al. Whispering gallery mode selection in optical bottle microresonators. Appl Phys Lett $2012 ; 100$

[62] Knight JC, Cheung G, Jacques F, et al. Phase-matched excitation of whispering-gallery-mode resonances by a fiber taper. Opt Lett 1997; 22: 1129-31

[63] Polynkin P, Polynkin A, Peyghambarian N, Mansuripur M. Evanescent field-based optical fiber sensing device for measuring the refractive index of liquids in microfluidic channels. Opt Lett 2005; 30: $1273-5$.

[64] Kude VP, Khairnar RS. Fabrication and numerical evaluation of the tapered single mode optical fiber: Detection of change in refractive index. Indian J Pure Ap Phy 2008; 46: 23-9.

[65] Sui CH, Wu PH, Ye BQ. Highly Sensitive Sensor for Detecting Refractive Index Change of Liquids Using Single Microfiber. Inec: 2010 3rd International Nanoelectronics Conference, Vols 1 and 2 2010: 1343-4. 
[66] Wang PF, Brambilla G, Ding M, Semenova Y, Wu Q, Farrell G. High-sensitivity, evanescent field refractometric sensor based on a tapered, multimode fiber interference. Opt Lett 2011; 36: 2233-5.

[67] Biazoli CR, Silva S, Franco MAR, Frazao O, Cordeiro CMB. Multimode interference tapered fiber refractive index sensors. Appl Optics 2012; 51: 5941-5.

[68] Qiu SJ, Chen Y, Kou JL, Xu F, Lu YQ. Miniature tapered photonic crystal fiber interferometer with enhanced sensitivity by acid microdroplets etching. Appl Optics 2011; 50: 4328-32.

[69] Liu Q, Wang Q. Refractive index sensor based on tapered PCF inline interferometer. Chin Opt Lett 2012; 10: 4.

[70] Li C, Qiu SJ, Chen Y, et al. Ultra-Sensitive Refractive Index Sensor With Slightly Tapered Photonic Crystal Fiber. IEEE Photonics Technol Lett 2012; 24: 1771-4.

[71] Monzon-Hernandez D, Villatoro J. High-resolution refractive index sensing by means of a multiple-peak surface plasmon resonance optical fiber sensor. Sens Actutors B-Chem 2006; 115: 227-31.

[72] Ma L, Matsuura Y. Surface Plasmon-Based Refractive Index Sensor Using a Tapered Bragg Fiber. Ofc: 2009 Conference on Optical Fiber Communication, Vols 1-5 2009: 1791-3.

[73] Cui Y, Shum PP, Wang GH, et al. Size Effect of Gold Nanoparticles on Optical Microfiber Refractive Index Sensors. 2011 Ieee Sensors 2011: 371-4.

[74] Liu GG, Wu YH, Li KW, et al. Mie Scattering-Enhanced FiberOptic Refractometer. IEEE Photonics Technol Lett 2012; 24: 65860.

[75] Lin HY, Huang CH, Cheng GL, et al. Tapered optical fiber sensor based on localized surface plasmon resonance. Opt Express 2012; 20: 21693-701.

[76] Grobnic D, Mihailov SJ, Ding HM, et al. Bragg grating evanescent field sensor made in biconical tapered fiber with femtosecond IR radiation. IEEE Photonics Technol Lett 2006; 18: 160-2.

[77] Fang X, Liao CR, Wang DN. Femtosecond laser fabricated fiber Bragg grating in microfiber for refractive index sensing. Opt Lett 2010; 35: 1007-9.

[78] Shao LY, Zhang AP, Liu WS, et al. Optical refractive-index sensor based on dual fiber-Bragg gratings interposed with a multimodefiber taper. IEEE Photonics Technol Lett 2007; 19: 30-2.

[79] Xu F, Brambilla G, Lu YQ. A microfluidic refractometric sensor based on gratings in optical fibre microwires. Opt Express 2009; 17: 20866-71.

[80] Zhang Y, Lin B, Tjin SC, et al. Refractive index sensing based on higher-order mode reflection of a microfiber Bragg grating. Opt Express 2010; 18: 26345-50.

[81] Ran Y, Tan YN, Sun LP, et al. 193nm excimer laser inscribed Bragg gratings in microfibers for refractive index sensing. Opt Express 2011; 19: 18577-83.

[82] Wang GG, Shum PP, Ho HP, et al. Modeling and analysis of localized biosensing and index sensing by introducing effective phase shift in microfiber Bragg grating (mu FBG). Opt Express 2011; 19: 8930-8.

[83] Liu YX, Meng C, Zhang AP, et al. Compact microfiber Bragg gratings with high-index contrast. Opt Lett 2011;36: 3115-7.

[84] Liang RB, Sun QZ, Wo JH, et al. Investigation on micro/nanofiber Bragg grating for refractive index sensing. Opt Commun 2012; 285: 1128-33.

[85] Ran Y, Jin L, Sun LP, et al. Bragg gratings in rectangular microfiber for temperature independent refractive index sensing. Opt Lett 2012; 37: 2649-51.

[86] Zhang JJ, Sun QZ, Liang RB, et al. Microfiber Fabry-Perot interferometer fabricated by taper-drawing technique and its application as a radio frequency interrogated refractive index sensor. Opt Lett 2012; 37: 2925-7.

[87] Cardenas-Sevilla GA, Monzon-Hernandez D, Torres-Gomez I, et al. Tapered Mach-Zehnder interferometer based on two mechanically induced long-period fiber gratings as refractive index sensor. Opt Laser Technol 2012; 44: 1516-20.

[88] Allsop T, Floreani F, Jedrzejewski K, et al. Refractive index sensing with long-period grating fabricated in biconical tapered fibre. Electron Lett 2005; 41: 471-2.

[89] Wu PH, Sui CH, Ye BQ. Modelling nanofiber Mach-Zehnder interferometers for refractive index sensors. J Modern Opt 2009; 56: 2335-9.

[90] Salceda-Delgado G, Monzon-Hernandez D, Martinez-Rios A, et al. Optical microfiber mode interferometer for temperatureindependent refractometric sensing. Opt Lett 2012; 37: 1974-6.
[91] Taghipoura Z, Zibaiia MI, Saeediana Z, et al. Enhanced sensitivity of gold modified tapered long period fiber grating for refractive index measurement. OFS 22nd International Conference on Optical Fiber Sensors 2012: 84213T

[92] Tan YZ, Sun LP, Jin L, et al. Microfiber Mach-Zehnder interferometer based on long period grating for sensing applications. Opt Express 2013; 21: 154-64.

[93] Li BY, Jiang L, Wang SM, et al. A new Mach-Zehnder interferometer in a thinned-cladding fiber fabricated by electric arc for high sensitivity refractive index sensing. Opt Laser Eng 2012; 50: 829-32.

[94] Yang J, Jiang L, Wang S, et al. High sensitivity of taper-based Mach-Zehnder interferometer embedded in a thinned optical fiber for refractive index sensing. Appl Opt 2011; 50: 5503-7.

[95] Chen NK, Yang TH, Feng ZZ, et al. Cellular-Dimension PicoliterVolume Index Microsensing Using Micro-Abrupt-Tapered Fiber Mach-Zehnder Interferometers. IEEE Photonics Technol Lett 2012; 24: 842-4.

[96] Wu D, Zhu T, Deng M, et al. Refractive index sensing based on Mach-Zehnder interferometer formed by three cascaded singlemode fiber tapers. Appl Opt 2011; 50: 1548-53.

[97] Wang P, Brambilla G, Ding M, et al. Enhanced Refractometer Based on Periodically Tapered Small Core Singlemode Fiber. IEEE Sens J 2013; 13: 180-5.

[98] Zhang S, Zhang WG, Geng PC, et al. Fiber Mach-Zehnder interferometer based on concatenated down- and up-tapers for refractive index sensing applications. Opt Commun 2013; 288: 47-51.

[99] Gao SC, Zhang WG, Geng PC, et al. Highly Sensitive In-Fiber Refractive Index Sensor Based on Down-Bitaper Seeded UpBitaper Pair. IEEE Photonics Technol Lett 2012; 24: 1878-81.

[100] Lu P, Harris J, Wang X, et al. Tapered-fiber-based refractive index sensor at an air/solution interface. Appl Opt 2012; 51: 7368-73.

[101] Lu P, Harris J, Xu Y, et al. Simultaneous refractive index and temperature measurements using a tapered bend-resistant fiber interferometer. Opt Lett 2012; 37: 4567-9.

[102] Lu P, Men LQ, Sooley K, et al. Tapered fiber Mach-Zehnder interferometer for simultaneous measurement of refractive index and temperature. Appl Phys Lett 2009; 94: 131110.

[103] Ji WB, Liu HH, Tjin SC, et al. Ultrahigh Sensitivity Refractive Index Sensor Based on Optical Microfiber. IEEE Photonics Technol Lett 2012; 24: 1872-4.

[104] Kou JL, Guo W, Xu F, et al. Highly Birefringent Optical-Fiberized Slot Waveguide for Miniature Polarimetric Interference Sensors: A Proposal. IEEE Sens J 2012; 12: 1681-5.

[105] Shi J, Xiao SL, Bi MH. Sensitivity-enhanced refractive index sensor by using tapered thin-core fiber based inline Mach-Zehnder interferometer. Opt Sens Biophoton Iii 2011; 8311.

[106] Yang WW, Zhang HF, Jia YR, et al. Hollow fiber liquid-phase microextraction for the determination of nimesulide in human plasma and its application to a pharmacokinetic study. Pharmazie 2011; 66: 564-9.

[107] Tai YH, Wei PK. Sensitive liquid refractive index sensors using tapered optical fiber tips. Opt Lett 2010; 35: 944-6.

[108] Kou JL, Feng J, Wang QJ, et al. Microfiber-probe-based ultrasmall interferometric sensor. Opt Lett 2010; 35: 2308-10.

[109] Kou JL, Qiu SJ, Xu F, et al. Miniaturized Metal-Dielectric-Hybrid Fiber Tip Grating for Refractive Index Sensing. IEEE Photonics Technol Lett 2011; 23: 1712-4.

[110] Zhu H, Wang YQ, Li BJ. Tunable Refractive Index Sensor with Ultracompact Structure Twisted by Poly(trimethylene terephthalate) Nanowires. ACS Nano 2009; 3: 3110-4.

[111] Liao CR, Wang DN, He XY, et al. Twisted Optical Microfibers for Refractive Index Sensing. IEEE Photonics Technol Lett 2011; 23 848-50.

[112] Lim KS, Aryanfar I, Chong WY, et al. Integrated Microfibre Device for Refractive Index and Temperature Sensing. Sensors-Basel 2012; 12: 11782-9.

[113] Layeghi A, Zibaii MI, Sadeghi J, et al. Sensing characteristics of tapered High-Birefringent optical fiber. OFS 22nd International Conference on Optical Fiber Sensors 2012: 84217O-1-4.

[114] Kang CJ, Han YG. Ambient index sensing technique using a Sagnac interferometer incorporating a tapered polarizationmaintaining fiber. OFS 22nd International Conference on Optical Fiber Sensors 2012: 84212Z-1-4. 
[115] L. B, Wang P, Semenova Y, et al. High sensitivity fiber refractometer based on an optical microfiber coupler. IEEE Photonics Technol Lett 2013; 25: 228-30.

[116] Shi L, Xu YH, Tan W, et al. Simulation of optical microfiber loop resonators for ambient refractive index sensing. Sensors-Basel 2007; 7: 689-96

[117] Xu F, Pruneri V, Finazzi V, et al. An embedded optical nanowire loop resonator refractometric sensor. Opt Express 2008; 16: 1062 7.

[118] Wang SS, Wang J, Li GX, et al. Modeling optical microfiber loops for seawater sensing. Appl Opt 2012; 51: 3017-23.

[119] Wang JW, Dai DX. Highly sensitive Si nanowire-based optical sensor using a Mach-Zehnder interferometer coupled microring. Opt Lett 2010; 35: 4229-31.

[120] Sun LP, Li J, Tan YZ, et al. Miniature highly-birefringent microfiber loop with extremely-high refractive index sensitivity. Opt Express 2012; 20: 10180-5.

[121] Pal SS, Mondal SK, Tiwari U, et al. Etched multimode microfiber knot-type loop interferometer refractive index sensor. Rev Sci Instrum 2011; 82:095107

[122] Xu F, Horak P, Brambilla G. Optical microfiber coil resonator refractometric sensor. Opt Express 2007; 15: 7888-93.

[123] White IM, Gohring J, Fan XD. SERS-based detection in an optofluidic ring resonator platform. Opt Express 2007; 15: 17433-42.

[124] Zamora V, Diez A, Andres MV, et al. Refractometric sensor based on whispering-gallery modes of thin capillaries. Opt Express 2007; 15: 12011-6.

[125] Corres JM, Bravo J, Matias IR, et al. Nonadiabatic tapered singlemode fiber coated with humidity sensitive nanofilms. IEEE Photonics Technol Lett 2006; 18: 935-7.

[126] Corres JM, Arregui FJ, Matias IR. Design of humidity sensors based on tapered optical fibers. J Lightwave Technol 2006; 24 : 4329-36

[127] Corres JM, Arregui FJ, Matias IR. Sensitivity optimization of tapered optical fiber humidity sensors by means of tuning the thickness of nanostructured sensitive coatings. Sens Actutors B-Chem 2007; 122: 442-9.

[128] Bariain C, Matias IR, Arregui FJ, et al. Optical fiber humidity sensor based on a tapered fiber coated with agarose gel. Sens Actutors B-Chem 2000; 69: 127-31.

[129] Zhang L, Gu FX, Lou JY, et al. Fast detection of humidity with a subwavelength-diameter fiber taper coated with gelatin film. Opt Express 2008; 16: 13349-53.

[130] Li T, Dong XY, Chan CC, et al. Humidity Sensor Based on a Multimode-Fiber Taper Coated With Polyvinyl Alcohol Interacting With a Fiber Bragg Grating. Ieee Sens J 2012; 12: 2205-8.

[131] Meng C, Xiao Y, Wang P, et al. Quantum-Dot-Doped Polymer Nanofibers for Optical Sensing. Advanced Materials 2011; 23: 3770-+.

[132] Li T, Zhao CL, Dong XY, et al. Relative Humidity Sensor Based on Photonic Crystal Fiber with Tapered and Filled in Polymer. Optical Sensors and Biophotonics Ii 2011; 7990: 288-9

[133] Wang P, Gu FX, Zhang L, et al. Polymer microfiber rings for highsensitivity optical humidity sensing. Appl Optics 2011; 50: G7G10.

[134] Wu Y, Zhang TH, Rao YJ, et al. Miniature interferometric humidity sensors based on silica/polymer microfiber knot resonators. Sens Actutors B-Chem 2011; 155: 258-63.

[135] Stiebeiner A, Rehband O, Garcia-Fernandez R, et al. Ultrasensitive fluorescence spectroscopy of isolated surface-adsorbed molecules using an optical nanofiber. Opt Express 2009; 17: 21704-11.

[136] Warken F, Vetsch E, Meschede D, et al. Ultra-sensitive surface absorption spectroscopy using sub-wavelength diameter optical fibers. Opt Express 2007; 15: 11952-8

[137] Takiguchi M, Yoshikawa Y, Yamamoto T, et al. Saturated absorption spectroscopy of acetylene molecules with an optical nanofiber. Opt Lett 2011; 36: 1254-6.

[138] Vishnoi G, Goel TC, Pillai PKC. Spectrophotometric studies of chemical species using tapered core multimode optical fiber. Sens Actutors B-Chem 1997; 45: 43-8.

[139] Zhang L, Wang P, Xiao Y, et al. Ultra-sensitive microfibre absorption detection in a microfluidic chip. Lab Chip 2011; 11: 3720-4.

[140] Wiejata PJ, Shankar PM, Mutharasan R. Fluorescent sensing using biconical tapers. Sens Actutors B-Chem 2003; 96: 315-20.
[141] Le Kien F, Dutta Gupta S, Balykin VI, et al. Spontaneous emission of a cesium atom near a nanofiber: Efficient coupling of light to guided modes Phys Rev A 2005; 72: 032509.

[142] Le Kien F, Hakuta K. Correlations between photons emitted by multiatom fluorescence into a nanofiber. Phys Rev A 2008; 77.

[143] Das M, Shirasaki A, Nayak KP, et al. Measurement of fluorescence emission spectrum of few strongly driven atoms using an optical nanofiber. Opt Express 2010; 18: 17154-64.

[144] Nayak KP, Das M, Le Kien F, et al. Spectroscopy of near-surface atoms using an optical nanofiber. Opt Commun 2012; 285: 4698704.

[145] Tian Y, Wang WH, Wu N, et al. Tapered Optical Fiber Sensor for Label-Free Detection of Biomolecules. Sensors-Basel 2011; 11: 3780-90.

[146] Minogin VG, Chormaic SN. Manifestation of the van der Waals Surface Interaction in the Spontaneous Emission of Atoms into an Optical Nanofiber. Laser Phys 2010; 20: 32-7.

[147] Nayak KP, Melentiev PN, Morinaga M, et al. Optical nanofiber as an efficient tool for manipulating and probing atomic fluorescence. Opt Express 2007; 15: 5431-8.

[148] Nayak KP, Hakuta K. Single atoms on an optical nanofibre. New Journal of Physics 2008; 10: 053003.

[149] Sague G, Vetsch E, Alt W, et al. Cold-atom physics using ultrathin optical fibers: Light-induced dipole forces and surface interactions. Phys Rev Lett 2007; 99: 163602.

[150] Russell L, Gleeson DA, Minogin VG, et al. Spectral distribution of atomic fluorescence coupled into an optical nanofibre. Journal of Physics B-Atomic Molecular and Optical Physics 2009; 42: 185006

[151] Wang SS, Pan XY, Tong LM. Modeling of nanoparticle-induced Rayleigh-Gans scattering for nanofiber optical sensing. Opt Commun 2007; 276: 293-7

[152] Baldini F, Ciaccheri L, Falai A, et al. Thymol blue immobilized on tapered fibres as an optical transducer for $\mathrm{pH}$ sensing. P Soc PhotoOpt Ins 1999; 3540: 28-33.

[153] Socorro AB, Del Villar I, Corres JM, et al. Tapered Single-Mode Optical Fiber pH Sensor Based on Lossy Mode Resonances Generated by a Polymeric Thin-Film. IEEE Sens J 2012; 12: 2598-603.

[154] Monzon-Hernandez D, Villatoro J, Talavera D, et al. Optical-fiber surface-plasmon resonance sensor with multiple resonance peaks. Appl Optics 2004; 43: 1216-20.

[155] Bagheria Z, Chenari Z, Karami Z, et al. Label free detection of quadruplex formation of peroxidase-like DNAzyme by tapered fiber optic biosensor. OFS 22nd International Conference on Optical Fiber Sensors 2012: 84215L-1-4.

[156] Saeediana Z, Zibaiia MI, Hosseinib SM, et al. Study of antibacterial effect of nanosilver particle by tapered optical fiber sensor. OFS 22nd International Conference on Optical Fiber Sensors 2012 84215K-1-4.

[157] Yao BC, Wu Y, Gong Y, et al. A Highly Sensitive and Fast Response Molecular Sensor Based on Graphene Coated Microfiber. OFS 22nd International Conference on Optical Fiber Sensors 2012: 842186-1-4.

[158] Vo-Dinh T. Nanobiosensors: Probing the sanctuary of individual living cells. J Cell Biochem 2002: 154-61

[159] McCulloch S, Uttamchandani D. Development of a fibre optic micro-optrode for intracellular $\mathrm{pH}$ measurements. Iee ProceedingsOptoelectronics 1997; 144: 162-7.

[160] Koranczi I, Reichert J, Ache HJ, et al. Submicron sensors for ion detection based on measurement of luminescence decay time. Sens Actutors B-Chem 2001; 74: 47-53.

[161] Scaffidi JP, Gregas MK, Seewaldt V, et al. SERS-based plasmonic nanobiosensing in single living cells. Anal Bioanal Chem 2009; 393: 1135-41.

[162] Zamarreno CR, Bravo J, Goicoechea J, et al. Response time enhancement of $\mathrm{pH}$ sensing films by means of hydrophilic nanostructured coatings. Sens Actutors B-Chem 2007; 128: 138-44.

[163] Waich K, Mayr T, Klimant I. Microsensors for detection of ammonia at ppb-concentration levels. Meas Sci Technol 2007; 18: 3195201.

[164] Barker SLR, Thorsrud BA, Kopelman R. Nitrite- and chlorideselective fluorescent nano-optodes and in in vitro application to rat conceptuses. Anal Chem1998; 70: 100-4.

[165] Barker SLR, Kopelman R, Meyer TE, et al. Fiber-optic nitric oxide-selective biosensors and nanosensors. Anal Chem 1998; 70 971-6. 
[166] Barker SLR, Kopelman R. Development and cellular applications of fiber optic nitric oxide sensors based on a gold-adsorbed fluorophore. Anal Chem 1998; 70: 4902-6.

[167] Song JM, Kasili PM, Griffin GD, et al. Detection of cytochrome c in a single cell using an optical nanobiosensor. Anal Chem 2004; 76: $2591-4$.

[168] Cordek J, Wang XW, Tan WH. Direct immobilization of glutamate dehydrogenase on optical fiber probes for ultrasensitive glutamate detection. Anal Chem 1999; 71: 1529-33.

[169] Cullum BM, Griffin GD, Miller GH, et al. Intracellular measurements in mammary carcinoma cells using fiber-optic nanosensors. Anal Biochem 2000; 277: 25-32.

[170] Kasili RM, Cullum BM, Griffin GD, et al. Nanosensor for in vivo measurement of the carcinogen benzo[a]pyrene in a single cell. $\mathbf{J}$ Nanosci Nanotechnol 2002; 2: 653-8.

[171] Kasili PM, Song JM, Vo-Dinh T. Optical sensor for the detection of caspase-9 activity in a single cell. J Am Chem Soc 2004; 126 : 2799-806.

[172] Liu XJ, Farmerie W, Schuster S, et al. Molecular beacons for DNA biosensors with micrometer to submicrometer dimensions. Anal Biochem 2000; 283: 56-63.

[173] Zheng XT, Li CM. Single living cell detection of telomerase overexpression for cancer detection by an optical fiber nanobiosensor. Biosens Bioelectron 2010; 25: 1548-52.

[174] Plaschke M, Geyer M, Reichert J, et al. Submicron fiber-optic sensors for calcium-ions and $\mathrm{pH}$ with internal calibration. Chem Biochem Environ Fiber Sens Ix 1997; 3105: 31-7.

[175] Harun SW, Jasim AA, Rahman HA, et al. Micro-Ball Lensed Fiber-Based Glucose Sensor. IEEE Photon J 2013; 13: 348-50.

[176] Lorenzi R, Jung YM, Brambilla G. In-line absorption sensor based on coiled optical microfiber. Appl Phys Lett 2011; 98: 173504.

[177] Sun YZ, Liu J, Frye-Mason G, Ja SJ, et al. Optofluidic ring resonator sensors for rapid DNT vapor detection. Analyst 2009; 134 : 1386-91.

[178] Sun YZ, Shopova SI, Frye-Mason G, et al. Rapid chemical-vapor sensing using optofluidic ring resonators. Opt Lett 2008; 33: 78890

[179] Vollmer F, Braun D, Libchaber A, et al. Protein detection by optical shift of a resonant microcavity. Appl Phys Lett 2002; 80: 4057 9.

[180] Vollmer F, Arnold S, Braun D, et al. Multiplexed DNA quanti. cation by spectroscopic shift of two microsphere cavities. Biophys J2003; 85: 1974-9.

[181] Vollmer F, Arnold S, Keng D. Single virus detection from the reactive shift of a whispering-gallery mode. Proc Nati Acad Sci USA 2008; 105: 20701-4.

[182] Ren HC, Vollmer F, Arnold S, et al. High-Q microsphere biosensor - analysis for adsorption of rodlike bacteria. Opt Express 2007; 15: $17410-23$

[183] Rosenzweig Z, Kopelman R. Development of a Submicrometer Optical-Fiber Oxygen Sensor. Anal Chem1995; 67: 2650-4.

[184] Villatoro J, Luna-Moreno D, Monzon-Hernandez D. Optical fiber hydrogen sensor for concentrations below the lower explosive limit. Sens Actutors B-Chem 2005; 110: 23-7.

[185] Villatoro J, Monzon-Hernandez D. Fast detection of hydrogen with nano fiber tapers coated with ultra thin palladium layers. Opt Express 2005; 13: 5087-92.

[186] Gu FX, Yin XF, Yu HK, et al. Polyaniline/polystyrene singlenanowire devices for highly selective optical detection of gas mixtures. Opt Express 2009; 17: 11230-5

[187] Monzon-Hernandez D, Luna-Moreno D, Escobar DM, et al. Optical microfibers decorated with $\mathrm{PdAu}$ nanoparticles for fast hydrogen sensing. Sens Actutors B-Chem 2010; 151: 219-22.

[188] Lee J, Kim J. Elastomeric microwire-based optical gas flowmeter with stretching-enabled tunability in measurement range. Opt Lett 2011; 36: 3789-91.

[189] Zhu S, Pang FF, Wang TY. Single-mode tapered optical fiber for temperature sensor based on multimode interference. Optic Sens Biophoton Iii 2011; 8311: 83112B.

[190] Veilleux C, Black RJ, Lapierre J, et al. Nematic Liquid-Crystal Clad Tapered Optical Fiber with Temperature Sensing Properties. J Appl Phys 1990; 67: 6648-53

[191] Zhang FJ, Wang B, Pang FF, et al. A luminescent temperature sensor based on a tapered optical fiber coated with quantum dots. Optic Sens Biophoton Ii 2011; 7990: 312-3
[192] Li Q, Wang SS, Chen YT, et al. Experimental Demonstration of Plasmon Propagation, Coupling, and Splitting in Silver Nanowire at 1550-nm Wavelength. IEEE J Select Top Quant Electron 2011; 17: $1107-11$.

[193] Zhang XB, Li JL, Li Y, et al. Sensing properties of intrinsic FabryPerot interferometers in fiber tapers. OFS 22nd International Conference on Optical Fiber Sensors 2012: 842189-1-4.

[194] André RM, Biazoli CR, Silva SO, et al. Strain-Temperature Discrimination using Multimode Interference in Tapered Fiber. IEEE Photonics Technol Lett 2013; 25: 155-8.

[195] Ji CK, Zhao CL, Kang J, et al. Multiplex and simultaneous measurement of displacement and temperature using tapered fiber and fiber Bragg grating. Rev Sci Instrum 2012; 83: 053109.

[196] Ding M, Wang PF, Brambilla G. A microfiber coupler tip thermometer. Opt Express 2012; 20: 5402-8.

[197] Kou JL, Feng J, Ye L, et al. Miniaturized fiber taper reflective interferometer for high temperature measurement. Opt Express 2010; 18: 14245-50

[198] Kou JL, Qiu SJ, Xu F, et al. Demonstration of a compact temperature sensor based on first-order Bragg grating in a tapered fiber probe. Opt Express 2011; 19: 18452-7.

[199] Feng J, Ding M, Kou JL, et al. An Optical Fiber Tip Micrograting Thermometer. IEEE Photon J 2011; 3: 810-4.

[200] Rajan G, Mathews S, Farrell G, et al. A liquid crystal coated tapered photonic crystal fiber interferometer. J Optics-Uk 2011; 13: 015403 .

[201] Semenova Y, Bo L, Mathews S, et al. Spectral tuning of a microfiber coupler with a liquid crystal overlay. OFS 22nd International Conference on Optical Fiber Sensors 2012: 842184-1-4.

[202] Harun SW, Lim KS, Damanhuri SSA, et al. Microfiber loop resonator based temperature sensor. Journal of the European Optical Society-Rapid Publications 2011; 6: 11026.

[203] Wu Y, Rao YJ, Chen YH, et al. Miniature fiber-optic temperature sensors based on silica/polymer microfiber knot resonators. Opt Express 2009; 17: 18142-7.

[204] Wu Y, Jia L, Zhang TH, et al. Microscopic multi-point temperature sensing based on microfiber double-knot resonators. Opt Commun 2012; 285: 2218-22.

[205] Zeng X, Wu Y, Hou CL, et al. A temperature sensor based on optical microfiber knot resonator. Opt Commun 2009; 282: 3817-9.

[206] Chen Y, Xu F, Lu YQ. Teflon-coated microfiber resonator with weak temperature dependence. Opt Express 2011; 19: 22923-8.

[207] Chen Y, Ming Y, Guo W, et al. Temperature Characteristics of Microfiber Coil Resonators Embedded in Teflon. Passive Components and Fiber-Based Devices VIII, Proc of SPIE-OSA-IEEE Asia Communications and Photonics 2012: 830711-1-4.

[208] Chen GY, Brambilla G, Newson TP. Inspection of Electrical Wires for Insulation Faults and Current Surges using Sliding Temperature Sensor based on Optical Microfiber Coil Resonator. Electron Lett 2013; 49: 46-7.

[209] Belal M, Song ZQ, Jung YM, et al. An interferometric current sensor based on optical fiber micro wires. Opt Express 2010; 18: 19951-6.

[210] Chen GY, Brambilla G, Newson TP. Spun optical microfiber. IEEE Photonics Technol Lett 2012; 24: 1663-6.

[211] Lim KS, Harun SW, Damanhuri SSA, et al. Current sensor based on microfiber knot resonator. Sens Actuators a-Physical 2011; 167 60-2.

[212] Chen GY, Brambilla G, Newson TP. Efficient Faraday rotation in birefringent optical microfibre loop resonators for current sensing. Electron Lett 2012; 48: 1547-8.

[213] Chen GY, Lee T, Ismaeel R, et al. Resonantly Enhanced Faraday Rotation in a Microcoil Current Sensor. IEEE Photonics Technol Lett 2012; 24: 860-3

[214] Chen GY, Lee T, Zhang XL, et al. Temperature compensation techniques for resonantly enhanced sensors and devices based on optical microcoil resonators. Opt Commun 2012; 285: 4677-83

[215] Sulaiman A, Harun SW, Aryangar I, et al. DC current sensing capability of microfibre Mach-Zehnder interferometer. Electron Lett 2012; 48: 943-5.

[216] Arregui FJ, Matias IR, Bariain C, et al. Experimental design rules for implementing biconically tapered singlemode optical fibre displacement sensors. P Soc Photo-Opt Ins 1998; 3483: 164-8.

[217] Luo HM, Li XW, Zou WW, et al. Temperature-Insensitive Microdisplacement Sensor Based on Locally Bent Microfiber Taper Modal Interferometer. IEEE Photon J 2012; 4: 772-8. 
[218] Yoon MS, Park S, Han YG. Simultaneous Measurement of Strain and Temperature by Using a Micro-Tapered Fiber Grating. J Lightw Technol 2012; 30: 1156-60.

[219] Martinez-Rios A, Monzon-Hernandez D, Torres-Gomez I, et al. An Intrinsic Fiber-Optic Single Loop Micro-Displacement Sensor. Sensors-Basel 2012; 12: 415-28.

[220] Jasim AA, Zulkifli AZ, Muhammad MZ, et al. A new compact micro-ball lens structure at the cleaved tip of microfiber coupler for displacement sensing. Sens Actuators A: Physical 2013; 189: 17781 .

[221] Monzon-Hernandez D, Martinez-Rios A, Torres-Gomez I, et al. Compact optical fiber curvature sensor based on concatenating two tapers. Opt Lett 2011; 36: 4380-2.

[222] Wang Y, Richardson D, Brambilla G, et al. Intensity measurement bend sensors based on periodically tapered soft glass fibers. Opt Lett $2011 ; 36: 558-60$.

[223] Zhang S, Zhang W, Gao S, et al. Fiber-optic bending vector sensor based on Mach-Zehnder interferometer exploiting lateral-offset and up-taper. Opt Lett 2012; 37: 4480-2.

[224] Wu ZF, Liu YG, Wang Z, et al. In-line Mach-Zehnder interferometer composed of microtaper and long-period grating in all-solid photonic bandgap fiber. Appl Phys Lett 2012; 101.

[225] Birks TA, Knight JC, Dimmick TE. High-resolution measurement of the fiber diameter variations using whispering gallery modes and no optical alignment. IEEE Photonics Technol Lett 2000; 12: 1823 .

[226] Sumetsky M, Dulashko Y, Fini JM, et al. Probing optical microfiber nonuniformities at nanoscale. Opt Lett 2006; 31: 2393-5.

[227] Sumetsky M, Dulashko Y. Radius variation of optical fibers with angstrom accuracy. Opt Lett 2010; 35: 4006-8.

[228] Lee CB, Kang JH, Y. JJ, et al. Phase locked loop based topography measurement of ultraprecision machined surface using the ball lensed and tapered fiber. OFS 22nd International Conference on Optical Fiber Sensors 2012: 8421B4-1-4.
[229] Chen GY, Zhang XL, Brambilla G, et al. Theoretical and experimental demonstrations of a microfiber-based flexural disc accelerometer. Opt Lett 2011; 36: 3669-71.

[230] Chen GY, Zhang XL, Brambilla G, et al. Enhanced responsivity of a flexural disc acceleration sensor based on optical microfiber. Opt Commun 2012; 285: 4709-14.

[231] Wang L, Liang PB, Liu ZH, et al. An optical trapping based microfiber vibration sensor. Proc Spie 2011; 7753: 77530F.

[232] Hou CL, Wu Y, Zeng X, et al. Novel high sensitivity accelerometer based on a microfiber loop resonator. Opt Eng 2010; 49: 014402.

[233] Wu Y, Zeng X, Rao YJ, et al. MOEMS Accelerometer Based on Microfiber Knot Resonator. IEEE Photonics Technol Lett 2009; 21: 1547-9.

[234] Wieduwilt T, Bruckner S, Bartelt H. High force measurement sensitivity with fiber Bragg gratings fabricated in uniform-waist fiber tapers. Meas Sci Technol 2011; 22: 075201.

[235] Wang X, Li W, Chen L, et al. Thermal and mechanical properties of tapered single mode fiber measured by OFDR and its application for high-sensitivity force measurement. Opt Express 2012; 20: 14779-88.

[236] Wang XZ, Li WH, Chen L, et al. High-sensitivity force measurement using optical tapered fiber with optical frequency-domain reflectometry. OFS 22nd International Conference on Optical Fiber Sensors 2012: 84211F-1-4.

[237] Digonnet MJF. Rotation Sensitivity of Gyroscopes Based on Distributed-Coupling Loop Resonators. J Lightw Technol 2011; 29: 3048-53.

[238] Xu B, Li Y, Sun M, Zhang ZW, et al. Acoustic vibration sensor based on nonadiabatic tapered fibers. Opt Lett 2012; 37: 4768-70.

[239] Chen GY, Brambilla G, Newson TP. Compact acoustic sensor based on air-backed mandrel coiled with optical microfiber. Opt Lett 2012; 37: 4720-2.

[240] Veilleux C, Lapierre J, Bures J. Liquid-Crystal-Clad Tapered Fibers. Opt Lett 1986; 11: 733-5.

[241] Ding H, Li X. All-fiber magnetic field sensor based on microfiber knot resonator and magnetic fluid. Opt Lett 2012; 37: 5187-9.

Received: July 30, 2012

(c) Chen et al.; Licensee Bentham Open.

This is an open access article licensed under the terms of the Creative Commons Attribution Non-Commercial License (http://creativecommons.org/licenses/by-nc/3.0/) which permits unrestricted, non-commercial use, distribution and reproduction in any medium, provided the work is properly cited. 